\title{
A Long Hard-X-Ray Look at the Dual Active Galactic Nuclei of M51 with NuSTAR
}

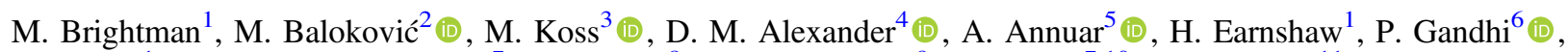

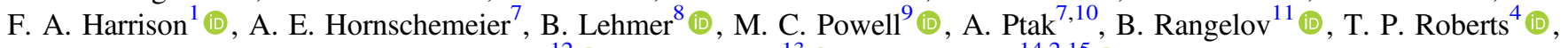 \\ D. Stern ${ }^{12}$ (D) D. J. Walton ${ }^{13}$ (D), and A. Zezas ${ }^{14,2,15}$ (D) \\ ${ }^{1}$ Cahill Center for Astrophysics, California Institute of Technology, 1216 East California Boulevard, Pasadena, CA 91125, USA \\ ${ }^{2}$ Harvard-Smithsonian Center for Astrophysics, 60 Garden Street, Cambridge, MA 02138, USA \\ ${ }^{3}$ Eureka Scientific, 2452 Delmer Street, Suite 100, Oakland, CA 94602-3017, USA \\ ${ }^{4}$ Department of Physics, Centre for Extragalactic Astronomy, Durham University, South Road, Durham DH1 3LE, UK \\ ${ }^{5}$ School of Applied Physics, Faculty of Science \& Technology, Universiti Kebangsaan Malaysia, 43600 Bangi, Selangor, Malaysia \\ ${ }^{6}$ School of Physics and Astronomy, University of Southampton, Highfield, Southampton SO17 1BJ, UK \\ ${ }^{7}$ NASA Goddard Space Flight Center, Code 662, Greenbelt, MD 20771, USA \\ ${ }^{8}$ Department of Physics, University of Arkansas, 226 Physics Building, 825 West Dickson Street, Fayetteville, AR 72701, USA \\ ${ }^{9}$ Yale Center for Astronomy and Astrophysics, and Physics Department, Yale University, P.O. Box 2018120, New Haven, CT 06520-8120, USA \\ ${ }^{10}$ Johns Hopkins University, Homewood Campus, Baltimore, MD 21218, USA \\ ${ }^{11}$ Department of Physics, Texas State University, 601 University Drive, San Marcos, TX 78666, USA \\ ${ }^{12}$ Jet Propulsion Laboratory, California Institute of Technology, Pasadena, CA 91109, USA \\ ${ }_{3}^{3}$ Institute of Astronomy, Madingley Road, Cambridge CB3 OHA, UK \\ ${ }^{14}$ Physics Department, University of Crete, 71003 Heraklion, Crete, Greece \\ ${ }^{15}$ Foundation for Research and Technology-Hellas (FORTH), Heraklion 71003, Greece \\ Received 2018 May 29; revised 2018 September 6; accepted 2018 September 12; published 2018 November 5
}

\begin{abstract}
We present a broadband X-ray spectral analysis of the M51 system, including the dual active galactic nuclei (AGNs) and several off-nuclear point sources. Using a deep observation by NuSTAR, new high-resolution coverage of M51b by Chandra, and the latest X-ray torus models, we measure the intrinsic X-ray luminosities of the AGNs in these galaxies. The AGN of M51a is found to be Compton-thick, and both AGNs have very low accretion rates $\left(\lambda_{\text {Edd }}<10^{-4}\right)$. The latter is surprising considering that the galaxies of M51 are in the process of merging, which is generally predicted to enhance nuclear activity. We find that the covering factor of the obscuring material in M51a is $0.26 \pm 0.03$, consistent with the local AGN obscured fraction at $L_{\mathrm{X}} \sim 10^{40} \mathrm{erg} \mathrm{s}^{-1}$. The substantial obscuring column does not support theories that the torus, presumed responsible for the obscuration, disappears at these low accretion luminosities. However, the obscuration may have resulted from the gas infall driven by the merger rather than the accretion process. We report on several extranuclear sources with $L_{\mathrm{X}}>10^{39} \mathrm{erg} \mathrm{s}^{-1}$ and find that a spectral turnover is present below $10 \mathrm{keV}$ in most such sources, in line with recent results on ultraluminous X-ray sources.
\end{abstract}

Key words: galaxies: active - galaxies: individual (M51) - galaxies: nuclei - X-rays: binaries - X-rays: galaxies

\section{Introduction}

M51, first cataloged by Messier (1781), consists of a pair of interacting galaxies: M51a (NGC 5194), a grand-design spiral galaxy, first to be classified as a spiral galaxy, and M51b (NGC 5195), a dwarf galaxy. The M51 galaxies are among the closest galaxies to our own at a distance of $8.58 \pm 0.10 \mathrm{Mpc}$, derived from the tip of the red giant branch method (McQuinn et al. 2016). As such, the galaxies have become a case study for the effects of galaxy interactions on galaxy evolution. The interaction is believed to be the cause of the distinctive spiral structure of M51a (Toomre \& Toomre 1972). Several works have investigated the interaction through $N$-body simulations. While it was originally thought that the two galaxies were experiencing their first close passage, later works have favored multiple past encounters, with one disk plane crossing 400-500 Myr ago and a more recent one 50-100 Myr ago, both at a separation of $\sim 25 \mathrm{kpc}$ (Salo \& Laurikainen 2000; Theis \& Spinneker 2003; Dobbs et al. 2010).

While interactions between galaxies have a large effect on their evolution, interactions are also predicted to increase the activity of their central supermassive black holes (SMBHs; e.g., Sanders et al. 1988; Hernquist 1989). This is due to the massive gas inflows caused by the resulting tidal forces, observational evidence for which has been found in large samples of galaxies (e.g., Ellison et al. 2011; Satyapal et al. 2014; Fu et al. 2018). The prediction is that the gas inflows into the nuclear regions also obscure the active nucleus (Hopkins et al. 2005), which has been revealed by recent results (Kocevski et al. 2015; Lanzuisi et al. 2015; Ricci et al. 2017a). Indeed, M51a and M51b are classed as a dual AGN: M51a hosts a Seyfert 2 in its nucleus (Stauffer 1982) that is obscured by Compton-thick (CT) material along the line of sight $\left(N_{\mathrm{H}}>1.5 \times 10^{24} \mathrm{~cm}^{-2}\right.$; Fukazawa et al. 2001). Although M51b is classified as a LINER in the optical (de Vaucouleurs et al. 1991), the Spitzer/ IRS detection of [Ne V] $\lambda 14.32 \mu$ m confirms that M51b is AGN powered (Goulding \& Alexander 2009). The AGN in M51b has been estimated to have an X-ray luminosity of $\sim 10^{39} \mathrm{erg} \mathrm{s}^{-1}$ in the $2-10 \mathrm{keV}$ band (Hernández-García et al. 2014). There is also evidence for AGN feedback from the nuclei of both galaxies (Querejeta et al. 2016; Schlegel et al. 2016), a major ingredient in the co-evolution of galaxies and their SMBHs.

The M51 system has been observed by all major X-ray observatories and was first detected in this band by the Einstein Observatory (Palumbo et al. 1985). Chandra was the first $\mathrm{X}$-ray observatory to resolve the nucleus of M51a and found it to have an iron $\mathrm{K} \alpha$ line with an equivalent width greater than $2 \mathrm{keV}$ (Terashima \& Wilson 2001; Levenson et al. 2002). The 
intrinsic X-ray luminosity has been estimated to be $4 \times 10^{40} \mathrm{erg} \mathrm{s}^{-1}$ (Xu et al. 2016) in the $2-10 \mathrm{keV}$ band, which makes it the lowest luminosity CTAGN known.

Besides detecting both nuclei, the Einstein Observatory found several ultraluminous X-ray sources (ULXs; see Kaaret et al. 2017; Roberts 2007 for reviews) associated with the galaxies. The ULX population was resolved into eight different sources by the high-resolution imager on ROSAT (Ehle et al. 1995). The ULXs have since been extensively cataloged and characterized by Liu \& Mirabel (2005), Dewangan et al. (2005), Winter et al. (2006), Swartz et al. (2011), Walton et al. (2011), and Kuntz et al. (2016). Kuntz et al. (2016) found that typically $\sim 5$ ULXs were active at any one time, with only two persistently active over 12 years of Chandra observations. The large number of ULXs in M51 is likely related to the high rates of star formation $\left(\sim 2.6 M_{\odot} \mathrm{yr}^{-1}\right.$; Schuster et al. 2007) that were triggered by the interaction between the galaxies (Smith et al. 2012). The ULXs in M51 show several interesting properties such as an intermediate-mass black hole (IMBH) candidate (Earnshaw et al. 2016) and two eclipsing ULXs (Urquhart \& Soria 2016), one that demonstrates apparent bimodal flux behavior that could indicate a pulsar in the propeller regime (Earnshaw et al. 2018) and one where a cyclotron resonance scattering feature has been detected, identifying the source as a neutron-star accretor (Brightman et al. 2018).

In this paper, we present a 210 ks observation of M51 with the Nuclear Spectroscopic Telescope Array (NuSTAR; Harrison et al. 2013) with the aim of characterizing the spectra of the nuclei of the two galaxies and the extranuclear point source population above $10 \mathrm{keV}$. While a short $18 \mathrm{ks}$ NuSTAR observation of M51 was already analyzed and presented by Xu et al. (2016), who studied the nucleus of M51a, and Earnshaw et al. (2016), who investigated one of the ULXs, the observation was too short to constrain spectral parameters well. In addition to the NuSTAR data, we present a $37.8 \mathrm{ks}$ Chandra ACIS-I observation that was taken contemporaneously and provides both soft X-ray coverage and the angular resolution to separate the crowded field. Furthermore, many Chandra ACIS-S observations already exist on M51 (Kuntz et al. 2016; Lehmer et al. 2017); these observations have all placed the nucleus of M51b at $\sim 4^{\prime}$ offaxis where the PSF is degraded and the source is near the edge of the detector or off it completely. Here we use ACIS-I with the aim point between the galaxies in order to better resolve the nucleus of M51b.

\section{Observational Data Reduction and Analysis}

The $37.8 \mathrm{ks}$ Chandra and $210 \mathrm{ks}$ NuSTAR observations studied here were taken contemporaneously between 2017 March 16-17. Table 1 provides a description of the observational data. In addition to these data, we use archival Chandra data taken during a large program with this observatory in 2012 in order to inform us of the long-term flux behavior of these sources (Kuntz et al. 2016). The details of these observations are listed in Table 1. The following sections describe the individual observations and data reduction. Spectral fitting was carried out using XSPEC v12.9.1 (Arnaud 1996), and all uncertainties quoted are at the $90 \%$ level.
Table 1

Observational Data

\begin{tabular}{lccr}
\hline \hline Observatory & ObsID & Start date (UT) & $\begin{array}{c}\text { Exposure } \\
(\mathrm{ks})\end{array}$ \\
\hline Chandra & 13813 & 2012 Sep 09 17:47:30 & 179.2 \\
Chandra & 13812 & 2012 Sep 12 18:23:50 & 179.2 \\
Chandra & 15496 & 2012 Sep 19 09:20:34 & 179.2 \\
Chandra & 13814 & 2012 Sep 20 07:21:42 & 179.2 \\
Chandra & 13815 & 2012 Sep 23 08:12:08 & 179.2 \\
Chandra & 13816 & 2012 Sep 26 05:11:40 & 179.2 \\
Chandra & 15553 & 2012 Oct 10 00:43:36 & 179.2 \\
NuSTAR & 60201062002 & 2017 Mar 16 15:21:09 & 47.2 \\
Chandra & 19522 & 2017 Mar 17 00:48:01 & 37.8 \\
NuSTAR & 60201062003 & 2017 Mar 17 16:56:09 & 163.1 \\
\hline
\end{tabular}

\subsection{Chandra}

The primary Chandra observation of M51 used here, ObsID 19522, was taken with ACIS-I at the optical axis. The aim point was placed between the two galaxies in order to improve upon the PSF size at the location of M51b, which in prior observations has largely been placed at large off-axis angles. We use these Chandra data to resolve the sources in the galaxy at $\sim \operatorname{arcsec}$ scales and to provide contemporaneous soft X-ray coverage to the NuSTAR data. The Chandra data were also used to inform the positions of the NuSTAR sources and were analyzed with CIAO v4.7.

No formal method is employed here to select the sources for which we conduct joint spectral fitting with $N u S T A R$. However, our informal method is to select sources that are bright in the 3-8 keV band as indicated by Chandra and that also show indications for emission in the NUSTAR 3-8 keV image. To do this, we first use the CIAO tool DMCOPY to create a $3-8 \mathrm{keV}$ Chandra image which we show in Figure 1. For clarity, we plot these sources as contours which represent sources with $>2$ counts pixel ${ }^{-1}$. Also plotted in Figure 1 with the same scale is the Spitzer/IRAC $3.6 \mu \mathrm{m}$ image for comparison.

\subsection{NuSTAR}

The raw NuSTAR data consist of two ObsIDs, 60201062002 and 60201062003, which have slightly different pointings and were reduced using the NUSTARDAS software package version 1.7.0. The events were cleaned and filtered using the nupipeline script with standard parameters. Due to higher-than-usual background during passages of the South Atlantic Anomaly (SAA), we filter the events files using saacalc $=1$ and saamode = strict. We also use XSELECT to make images in the $3-8 \mathrm{keV}$ and the $8-24 \mathrm{keV}$ bands and use XIMAGE to co-add the images from the two ObsIDs and the two focal plane modules (FPMs). Based on the positions of the brightest sources, we found an astrometric offset of 8 " 5 between the Chandra and NuSTAR positions. The offset was $\Delta$ R.A. $=7 "$. 4 and $\Delta$ decl. $=4 ! " 3$, which are typical values for astrometric offsets between Chandra and NuSTAR (Lansbury et al. 2017). We show the astrometrically corrected images in Figure 1.

Figure 1 shows that NUSTAR finds $\sim 8$ sources of emission within the galaxies of M51 in the $3-8 \mathrm{keV}$ band, where for some of which the PSFs are overlapping. Furthermore, the high-resolution Chandra data show that for at least two NUSTAR sources, more than one source contributes significantly to the NuSTAR PSF. We select these eight sources for 


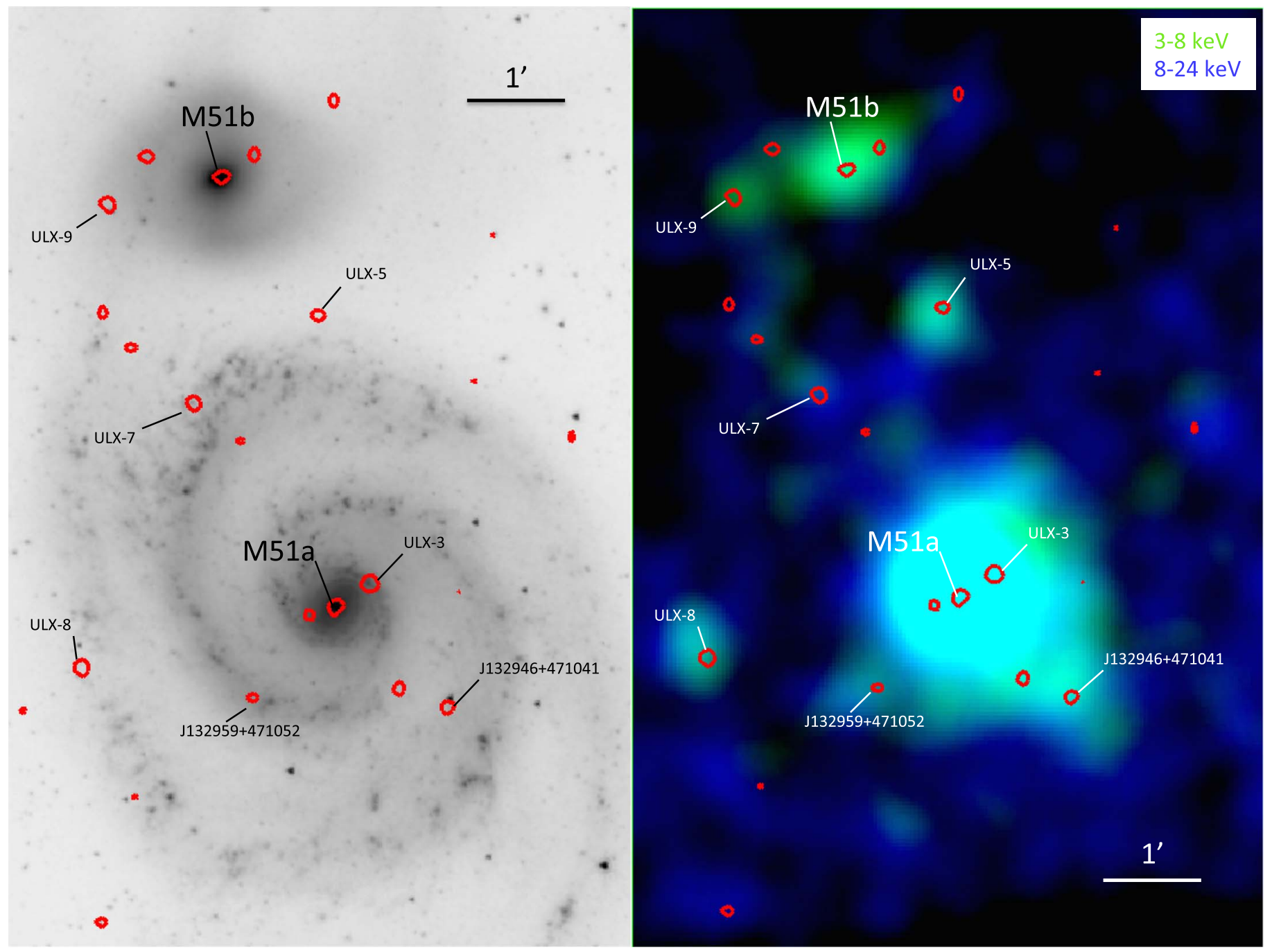

Figure 1. Multiband images of M51. The panel on the left shows Spitzer/IRAC $3.6 \mu \mathrm{m}$ image, overlaid with Chandra 3-8 keV contours, showing the brightest sources $\left(>2\right.$ counts pixel $^{-1}$ ), in red. The right panel shows the same contours overlaid on the NUSTAR 3-8 keV image in green and the NuSTAR 8-24 keV image in blue. The NUSTAR images, which consist of co-added FPMA+B images, have been smoothed with a $10^{\prime \prime}$ kernel.

spectral analysis and conduct joint spectral fitting of the sources where Chandra resolved more than one source. Approximately, these sources are selected with $3-8 \mathrm{keV}$ fluxes $>10^{-14} \mathrm{erg} \mathrm{cm}^{-2} \mathrm{~s}^{-1}$. From Poisson statistics, we find that all sources are significantly detected in the $0.5-8 \mathrm{keV}$ Chandra and 3-30 keV NuSTAR bands, with the probabilities of background fluctuations being $\ll 10^{-10}$.

We use the nuproducts task to generate the spectra and the corresponding response files for each ObsID separately. For the brightest NuSTAR source, the nucleus of M51a, we extract spectra using a $40^{\prime \prime}$ circular region, which corresponds to an encircled energy fraction of $\sim 55 \%$ (Harrison et al. 2013; Madsen et al. 2015). This extraction region includes ULX3, so we fit the spectra of these two sources jointly. For the rest of the NUSTAR sources, which are fainter, we use $20^{\prime \prime}$ circular regions in order to reduce the background and avoid overlapping PSFs. Twenty arcsec encloses around $30 \%$ of the counts. Despite this, the extraction region for the nucleus of M51b includes at least one other source that contributes significantly to the NUSTAR PSF, so we model the spectra of these sources jointly. Background spectra were extracted from $50^{\prime \prime}$ circular regions on the same detector as the respective source, taking care to avoid detected sources, including those not evident in the
NUSTAR image but shown to be bright in the $3-8 \mathrm{keV}$ band by Chandra. For each source, the spectral data from FPMA for each observation were co-added to each other using the HEASOFT tool ADDSPEC. The same was done for the data from FPMB. The data from FPMA and FPMB were not co-added to each other, but used for simultaneous fitting instead. The resulting exposure after background filtering and co-adding is 193.8 and 193.3 ks for FPMA and FPMB, respectively.

\subsection{Palomar}

Optical spectroscopic observations of the nucleus of M51a were taken as part of the BAT AGN Spectroscopic Survey (BASS) follow-up of newly detected 105 month Swift/BATdetected AGNs (Oh et al. 2018). The aim was to determine the mass of the SMBH from the stellar velocity dispersion. The observation took place at UT 2018 March 27 with the Palomar Double Spectrograph (DBSP) on the Hale 200-inch telescope for $600 \mathrm{~s}$ using the 1200 lines/mm grating. We used the $2^{\prime \prime}$ slit at the parallactic angle $\left(160^{\circ}\right)$ and extracted a nuclear aperture of $10^{\prime \prime}$. We measured the sky lines to have an FWHM $=2.4 \AA$ at $5000 \AA$ and $\mathrm{FWHM}=2.1 \AA$ at $8500 \AA$ corresponding to an 
Table 2

X-Ray Count Rates for All Sources in M51 Analyzed Here

\begin{tabular}{lccc}
\hline \hline Source & ACIS & FPMA & FPMB \\
& $0.5-8 \mathrm{keV}$ & $3-30 \mathrm{keV}$ & $3-30 \mathrm{keV}$ \\
\hline M51a nucleus & $3.5 \pm 0.1$ & $7.7 \pm 0.2$ & $7.5 \pm 0.2$ \\
ULX3 & $3.7 \pm 0.3$ & & \\
M51b nucleus & $2.2 \pm 0.2$ & $0.5 \pm 0.1$ & $0.7 \pm 0.1$ \\
Extranuclear source & $0.8 \pm 0.2$ & & \\
ULX5 & $2.6 \pm 0.3$ & $0.6 \pm 0.1$ & $0.5 \pm 0.1$ \\
ULX7 & $4.4 \pm 0.3$ & $0.2 \pm 0.1$ & $0.3 \pm 0.1$ \\
ULX8 & $8.3 \pm 0.5$ & $0.5 \pm 0.1$ & $0.5 \pm 0.1$ \\
ULX9 & $5.7 \pm 0.4$ & $0.4 \pm 0.1$ & $0.3 \pm 0.1$ \\
J132946+471041 & $3.6 \pm 0.3$ & $0.7 \pm 0.1$ & $0.4 \pm 0.1$ \\
J132959+471052 & $1.9 \pm 0.2$ & $0.4 \pm 0.1$ & $0.3 \pm 0.1$ \\
\hline
\end{tabular}

Note. Background-subtracted source count rates $\left(\right.$ counts ks ${ }^{-1}$ ) in the Chandra ACIS-I, NUSTAR FPMA, and NUSTAR FPMB detectors.

instrumental limit of $64 \mathrm{~km} \mathrm{~s}^{-1}$ and $30 \mathrm{~km} \mathrm{~s}^{-1}$ respectively, at the redshift of M51.

The velocity dispersion was measured using the penalized PiXel Fitting software (pPXF; Cappellari \& Emsellem 2004; Cappellari 2017) version 5.0 to fit with the optimal stellar templates. We used 86 stars from The X-Shooter Spectral Library of stellar spectra (Chen et al. 2014) with $R=10,000$, which cover $3000-25000 \AA$. These templates have been observed at a higher spectral resolution than the AGN observations and are convolved in PPXF to the spectral resolution of each observation before fitting. When fitting the stellar templates, all of the prominent emission lines were masked. After the best-fitting stellar template was removed, the residual emission lines were fit. We found a velocity dispersion of $75 \pm 4 \mathrm{~km} \mathrm{~s}^{-1}$ when fitting the $3950 \AA$ to the $5500 \AA$, which includes the $\mathrm{CaH}+\mathrm{K}$ and $\mathrm{Mg}$ I regions and $63 \pm 4 \mathrm{~km} \mathrm{~s}^{-1}$ when fitting the calcium triplet region (8350-8900 $\mathrm{A}$ ). More details on the reductions and pPXF fitting can be found in Koss et al. 2017. We note that both these measurements are significantly below the previous literature value $\left(102 \mathrm{~km} \mathrm{~s}^{-1}\right.$; Nelson \& Whittle 1995), most likely due to the uncertainties in subtracting the instrumental resolution of lower resolution observations.

\section{Spectral Fitting}

We carry out X-ray spectral fitting on the eight brightest NUSTAR sources in M51: the nucleus of M51a, which includes the emission from ULX3; the nucleus of M51b, which includes emission from a bright extranuclear source; and six additional off-nuclear sources. We describe the spectral fitting procedures for each source individually in the following subsections. The count rate for each source from each detector is shown in Table 2.

For the bright nucleus of M51a, we group both the Chandra and NUSTAR data with a minimum of 20 counts. We carry out spectral fitting with background-subtracted spectra and use the $\chi^{2}$ statistic as the fit statistic. The NuSTAR data remain sourcedominated up to $\sim 30 \mathrm{keV}$, but we consider the entire 3-79 keV NUSTAR band for spectral fitting. We carry out spectral fitting over the $0.5-8 \mathrm{keV}$ band for the Chandra data.

Since many archival Chandra observations of M51 are available in addition to the one simultaneously taken with NuSTAR, we investigate the long-term flux behavior of our sources. Specifically, we study a period in 2012 where many of

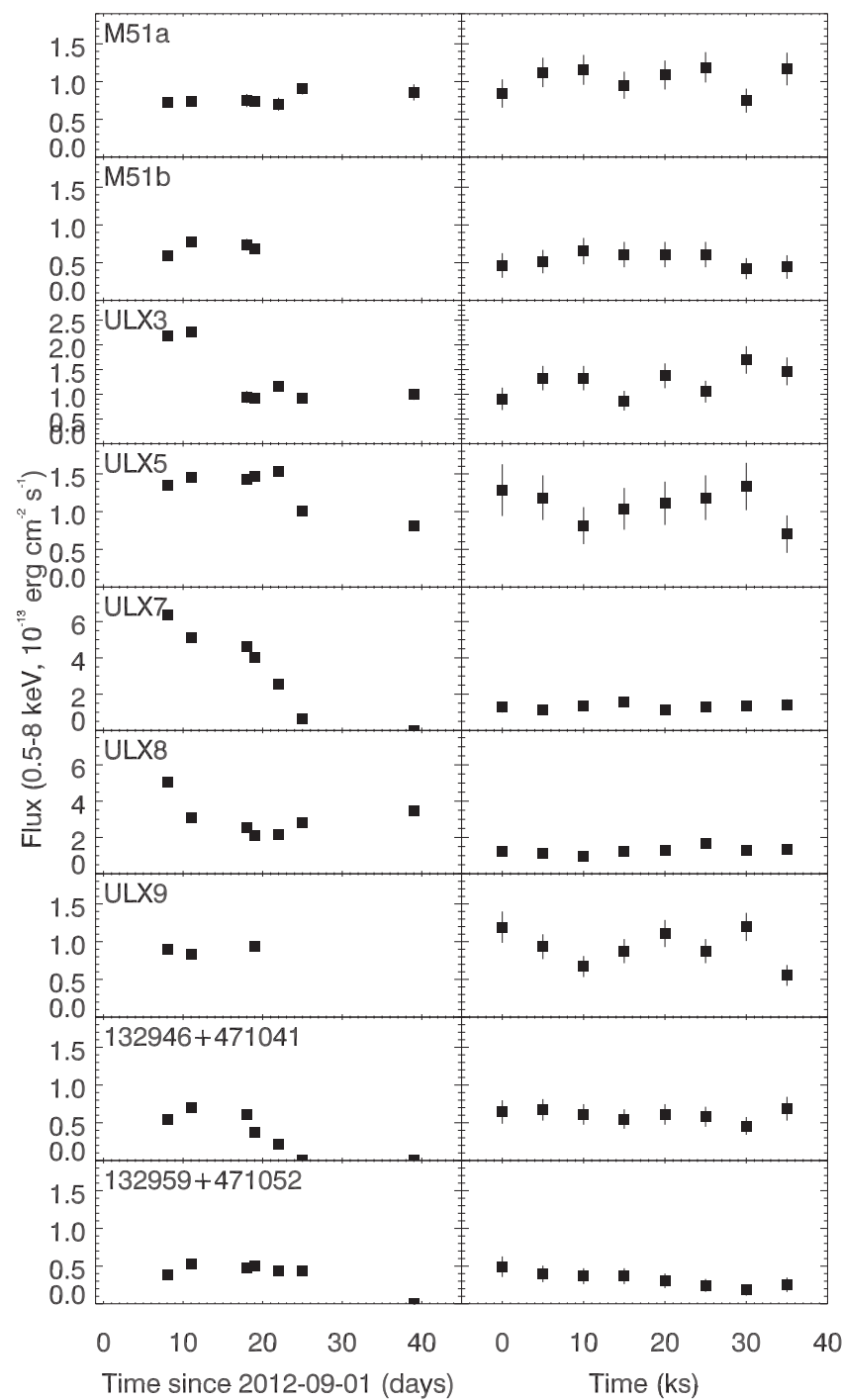

Figure 2. Long-term (left) and short-term (right) Chandra light curves of the sources studied here. Fluxes in the $0.5-8 \mathrm{keV}$ band are converted from count rates assuming the best-fit spectral models.

these observations were taken over a period of $\sim 40$ days. We also examine the variability of each source during the latest Chandra observation. For this, we convert the backgroundsubtracted count rates into $0.5-8 \mathrm{keV}$ fluxes assuming the spectral models described in the following sections. We plot the long- and short-term light curves in Figure 2.

The long-term $0.5-8 \mathrm{keV}$ flux of the M51a nucleus remains approximately constant, varying by $\pm 20 \%$ around the mean. Since it has not varied considerably over the years, we utilize all existing Chandra data on the nucleus of M51a. These are ObsIDs 13812, 13813, 13814, 13815, 13816, 15496, 15553, and 19522, totaling $783 \mathrm{ks}$ of exposure. We fit the Chandra data and the NUSTAR data simultaneously using crosscalibration constants, $C_{\mathrm{ACIS}}, C_{\mathrm{FPMA}}$, and $C_{\mathrm{FPMB}}$, to account for the differing instrumental responses. $C_{\mathrm{FPMA}}$ is fixed to unity while the others are free to vary.

For the nucleus of M51b, the differing off-axis angles and PSF sizes that have resulted from these in the previous observations make assessing the variability challenging so we do not co-add the Chandra data of M51b. Most of the ULXs show long-term flux variability. Therefore, for these sources, 
we only use the latest Chandra ObsID 19522, which was simultaneous with $N u S T A R$. Also, due to their low-count nature, we only lightly group the spectra, with a minimum of 1 count for Chandra and 3 counts for NuSTAR (see Lanzuisi et al. 2013 for an investigation into the grouping of spectra with a low number of counts per bin). We use the Cash statistic (Cash 1979) with background-subtracted spectra. While the use of the Cash statistic cannot be strictly used in the case where the background has been subtracted, XSPEC implements a modified version of the Cash statistic to account for this, known as the W-statistic. ${ }^{16} \mathrm{We}$ only consider NUSTAR data up to $30 \mathrm{keV}$, beyond which the photon statistics are poor. For these sources, we fix all cross-calibration constants to unity unless we find evidence for a significant deviation from this.

\subsection{The Compton-thick Nucleus of M51a}

The nucleus of M51a is well known to be Compton-thick, that is, the optical depth to Compton scattering of X-rays off electrons is greater than unity. Therefore, the effect of Compton scattering must be taken into account when modeling the X-ray spectra of these sources. Several X-ray spectral models that have been compiled especially for this reason now exist. These models take an intrinsic AGN power-law spectrum and subject it to photoelectric absorption, Compton scattering, and iron fluorescence using Monte Carlo simulations, assuming a toroidal obscuring structure thought to exist in the inner regions of the AGN.

We use the most up-to-date X-ray spectral torus models in our analysis, the mytorus model (Murphy \& Yaqoob 2009) and the borus ${ }^{17}$ model (Baloković et al. 2018), which is an update to the torus model (Brightman \& Nandra 2011a). These models differ mostly in the geometry of the toroidal structure assumed. Both assume a smooth axisymmetric structure, but for mytorus, the torus has a circular cross section, whereas the borus model is based on a sphere with a biconical cutout. In this way, for lines of sight through the torus, the line-of-sight $-N_{\mathrm{H}}$ in the mytorus is inclination dependent, but not for the borus model. For both models, the direct transmitted component, which is composed of photons that travel through the structure without undergoing interactions, can be decoupled from the scattered component, where the photons have Compton scattered off the obscuring material. This gives the models greater freedom, for example, to emulate different geometries such as a clumpy distribution of gas, but also adds degeneracies. For X-ray spectral fitting of absorbed sources, there is a degeneracy between the X-ray spectral index, $\Gamma$, and the column density, $N_{\mathrm{H}}$, especially in low-quality spectra over a narrow band. This degeneracy is mostly mitigated using these torus models that include the fluorescent lines and when high-quality broadband spectra are used, such as what we have done here. For the models with the covering factor as a free parameter, this can be degenerate with the $N_{\mathrm{H}}$, but again this mostly affects lower signal-to-noise data (e.g., Baloković et al. 2018).

For spectral fitting of the nucleus of M51a, we use the coadded Chandra spectra extracted and merged with ACIS EXTRACT (Broos et al. 2010). We only consider emission from within $1^{\prime \prime}$ of the central point source, corresponding to an

\footnotetext{
16 https://heasarc.gsfc.nasa.gov/xanadu/xspec/manual/XSappendixStatistics. html

${ }^{17}$ The specific geometry and version number used is borus02_afe1_v170227a.fits.
}

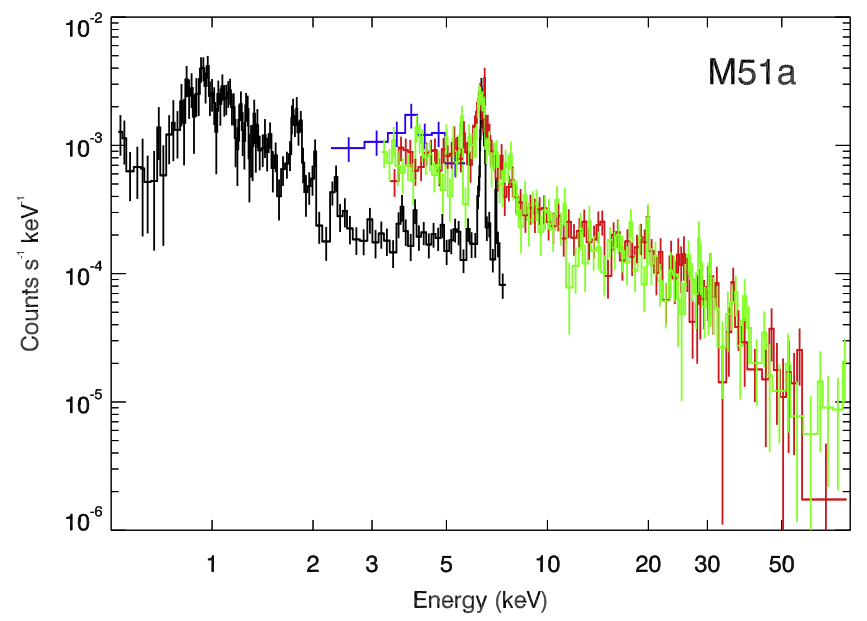

Figure 3. Chandra (the nucleus in black and ULX3 in blue) and NuSTAR (FPMA in red and FPMB in green) spectra of the M51a nuclear region folded through the instrumental responses and rebinned for clarity.

encircled energy fraction of $90 \%$, and ignore the extended emission surrounding the nucleus since this does not contribute in the NUSTAR band above $3 \mathrm{keV}$ as seen in the $3-8 \mathrm{keV}$ Chandra image (Figure 1). See $\mathrm{Xu}$ et al. (2016) for a description of the soft extended emission. The $40^{\prime \prime}$ region used to extract the NuSTAR data also includes two hard point sources seen in the Chandra images. One of these is ULX3, the Chandra spectrum of which we include in our spectral analysis. We model the spectrum of ULX3 with an absorbed cutoff power law. The other hard source is not bright enough to contribute significantly to the NuSTAR spectrum, with less than one-third the count rate of the nucleus or ULX3 in the $3-8 \mathrm{keV}$ band, so we ignore it in spectral fitting. The spectral data are shown in Figure 3.

Despite only extracting spectra from within the central $1^{\prime \prime}$, which corresponds to $\sim 40 \mathrm{pc}$ at $8.5 \mathrm{Mpc}$, excess soft X-ray emission is seen in the Chandra spectrum above the characteristic reflection spectrum. This likely arises from scattered nuclear light and emission from gas photoionized by the AGN. In order to avoid the complexities associated with this emission, we consider only counts above $3 \mathrm{keV}$.

We find that mytorus in coupled mode $\left(N_{\mathrm{H}}, \Gamma\right.$, inclination, and normalization of the transmitted component and scattered component tied to each other) provides a fit to the data with $\chi^{2}=399.6$ with 258 degrees of freedom (DoF). In order to find a possible better fit, we decouple the inclination of the transmitted component and the scattered component, which improves the fit statistic to $\chi^{2} / \operatorname{DoF}=374 / 257$. We then decouple the $N_{\mathrm{H}}$ parameter of the two components, which leads to an improvement of the fit statistic to $\chi^{2} / \mathrm{DoF}=356.9 / 256$. Finally, we decouple the normalizations, which leaves the two components fully decoupled; however, this actually worsens the fit $\left(\chi^{2} / \mathrm{DoF}=363.0 / 255\right)$. We therefore declare our bestfit mytorus model to be the one where only the inclination and $N_{\mathrm{H}}$ parameters of the transmitted and scattered components are decoupled, with $\chi^{2} / \mathrm{DoF}=356.9 / 256$.

For the borus model, we start by constraining the inclination of the scattered component to be greater than the opening angle of the torus (such that the line of sight is through the torus) to be fully self consistent. This provides a fit to the data with $\chi^{2}=359.3$ with 258 DoF. However, if we allow the full range of inclination angles for the scattered component, we 


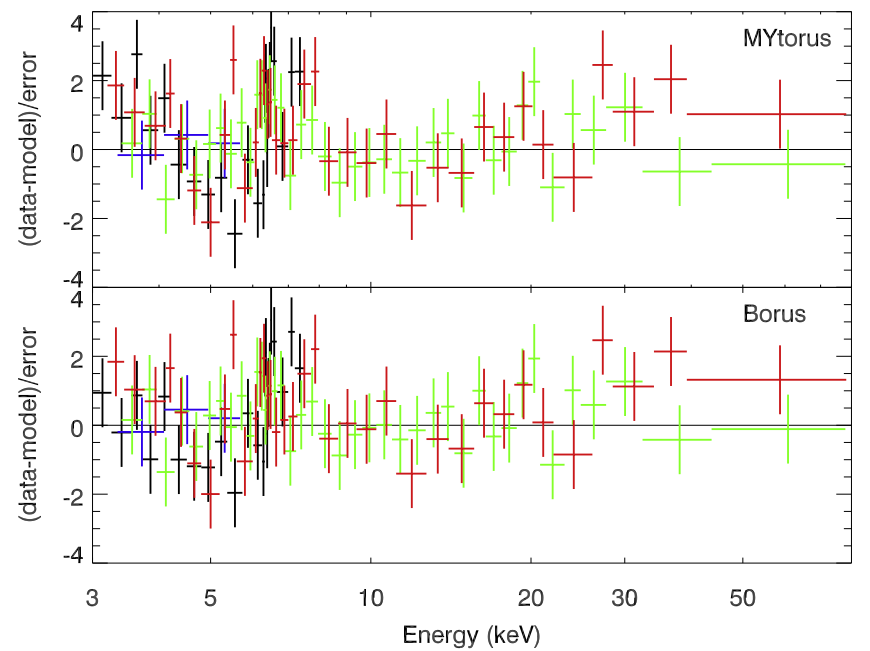

Figure 4. Chandra (the nucleus in black and ULX 3 in blue) and NuSTAR (FPMA in red and FPMB in green) residuals to the fits with the mytorus and borus models. Included in both fits is the cutoffpl model for ULX3.

find that the data prefer a small, close to face-on view of the torus for the scattered component, improving the fit significantly to $\chi^{2} / \mathrm{DoF}=315.7 / 258$. This was similarly the case for the mytorus model and implies that the observed scattered component is stronger than the simple geometries the torus models can account for. Decoupling the $N_{\mathrm{H}}$ parameter of the borus model does not improve the fit $\left(\chi^{2} / \mathrm{DoF}=318.4\right.$ / 256). The same is true when decoupling the normalization parameter $\left(\chi^{2} / \mathrm{DoF}=318.4 / 255\right)$. We therefore declare our best-fit borus model to be the one where only the full range of inclination angles for the scattered component is allowed, with $\chi^{2} / \mathrm{DoF}=315.7 / 258$.

We show the broadband residuals to the fit with both the best-fit mytorus and borus models in Figure 4. These residuals do not show any obvious deviations with the exception of the Fe-K complex around $6.4 \mathrm{keV}$. We show a zoom-in of the $5-8 \mathrm{keV}$ band, which contains the $\mathrm{Fe}-\mathrm{K}$ complex in Figure 5. Considering the spectral data in this energy band alone, $\chi^{2} / \mathrm{DoF}=156.6 / 64$ for mytorus and $\chi^{2} / \mathrm{DoF}=133.0 / 66$ for borus, which shows that neither model fits the $\mathrm{Fe} \mathrm{K} \alpha$ line well, although borus provides a slightly better fit.

The equivalent width (EW) of the $\mathrm{Fe} \mathrm{K} \alpha$ line of M51a is known to be one of the highest measured (Terashima \& Wilson 2001; Levenson et al. 2002). The torus models that we utilize here treat the $\mathrm{Fe} \mathrm{K} \alpha$ and continuum self-consistently and as such, the $\mathrm{Fe} \mathrm{K} \alpha$ line plays a part in constraining the torus geometry. Since the $\mathrm{Fe} \mathrm{K} \alpha$ line is not treated as a separate component in our fits, a direct measurement of the EW is not given from these models. However, for mytorus, the line component can be decoupled from the continuum. We measure the $\mathrm{EW}$ of the $\mathrm{Fe} \mathrm{K} \alpha$ line by decoupling the normalization of the line component in mytorus, which yields $4.1 \mathrm{keV}$. This is for the $\mathrm{Fe} \mathrm{K} \alpha$ line; however, this still includes constraints from the $\mathrm{Fe} \mathrm{K} \beta$ line in the fit, which cannot be removed from the spectral fit. We then remove the fluorescent lines altogether from the mytorus fit and add a single Gaussian component at $6.4 \mathrm{keV}$ to model the $\mathrm{Fe} \mathrm{K} \alpha$ emission. For this Gaussian component, we measure $E=6.41 \pm 0.01 \mathrm{keV}, \sigma=51_{-13}^{+12} \mathrm{eV}$, and $\mathrm{EW}=3.3_{-0.46}^{+0.27} \mathrm{keV}$. The lower $\mathrm{EW}$ measured by the Gaussian with respect to mytorus may be due to the $\mathrm{Fe} \mathrm{K} \beta$

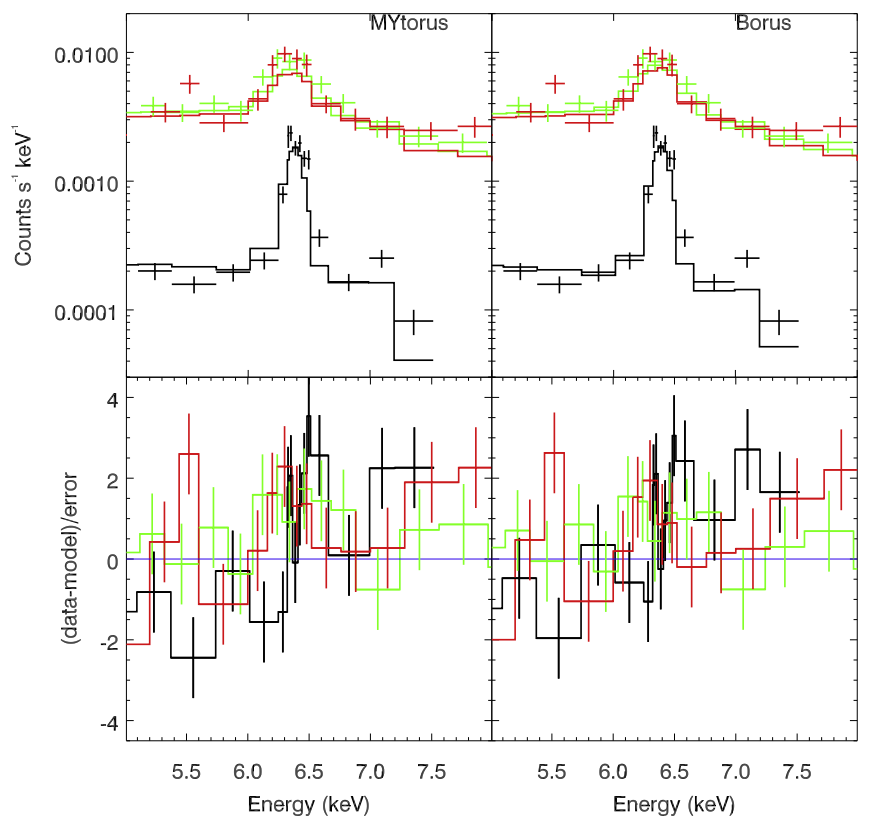

Figure 5. Top-Chandra (black) and NUSTAR (FPMA in red and FPMB in green) spectra of the M51a nucleus around the $\mathrm{Fe} \mathrm{K} \alpha$ emission line. The NUSTAR data have been multiplied by a factor of 4 for plotting clarity. Bottom - data to model ratios.

line being stronger with respect to the $\mathrm{Fe} \mathrm{K} \alpha$ line than the model expectation.

For the borus model of the nucleus, $C_{\mathrm{ACIS}} / C_{\mathrm{FPMA}}=$ $0.84_{-0.06}^{+0.12}$, and for the cutoffpl model of the ULX, $C_{\mathrm{ACIS}} / C_{\mathrm{FPMA}}=0.69_{-0.11}^{+0.12}$. While the cross-calibration constant for the nucleus is almost consistent with unity, the one for the ULX is not. The Chandra light curve of the ULX shows that it is rising in flux (Figure 2), which it may have continued to do during the latter part of the NUSTAR observation when Chandra was no longer observing. This would explain the apparent greater contribution of the ULX to the NuSTAR spectrum than Chandra shows. Similar cross-calibration constants result from the mytorus model.

We show the best-fit borus model of the nucleus and the cutoffpl model of ULX3 in Figure 6. We list the best-fit parameters for both the best mytorus and borus model fits to the nucleus in Table 3 . We present best-fit parameters for ULX3 in Section 3.3 along with the other extranuclear sources. The $N_{\mathrm{H}}$ of the nucleus measured by the two torus models agree, showing that the source is heavily Compton-thick (although mytorus does not probe $\left.\log \left(N_{\mathrm{H}} / \mathrm{cm}^{-2}\right)>25\right)$. Both models also indicate that the intrinsic photon index is low, $\Gamma=1.4-1.8$ (the hard lower limit on both models is 1.4). The AGN is estimated to have an intrinsic $2-10 \mathrm{keV}$ X-ray luminosity of $2 \times 10^{40} \mathrm{erg} \mathrm{s}^{-1}$ from both models. The borus model also estimates a low covering factor of the torus of $0.26 \pm 0.03$.

\subsection{The LINER Nucleus of M51b}

Upon examination of the new Chandra data, with its improved resolution of the M51b nuclear region, we noted several X-ray point sources that could be identified as the nucleus. In order to identify the true nuclear source, we investigated data from longer wavelengths, specifically high-resolution $H S T /$ WFC3 data. We present the multiwavelength images in Figure 7 . The nucleus is evident as a strong centrally peaked near-infrared (NIR) source, whereas in the near-ultraviolet, the nuclear region is more 


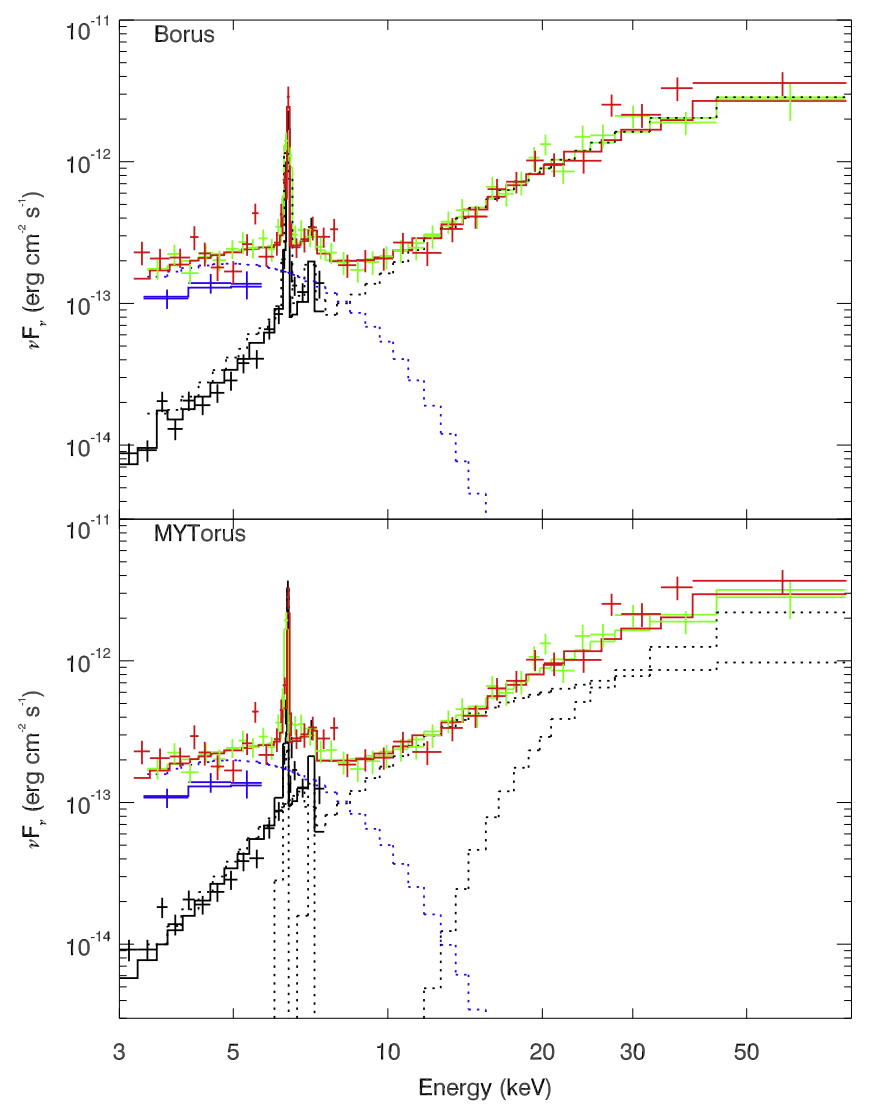

Figure 6. Chandra (the nucleus in and ULX3 in blue) and NUSTAR (FPMA in red and FPMB in green) spectra of the M51a nuclear region unfolded through the instrumental responses assuming the best-fit borus model (top, black dotted line) and mytorus model (bottom, black dotted line showing transmitted, scattered, and line components) for the nucleus and cutoffpl model for ULX3 (blue dotted line).

extended. We use the position of the NIR source to inform us which is the nuclear X-ray source. Recent results from Rampadarath et al. (2018) using high-resolution $\left(<1^{\prime \prime}, \sim 10 \mathrm{pc}\right)$ e-MERLIN L (1-2 GHz) and $C$-band $(4-8 \mathrm{GHz})$ radio data identify the same X-ray source as the nucleus.

We extract the Chandra spectra from the nucleus and the brightest extranuclear X-ray source within the $20^{\prime \prime}$ radius of the NUSTAR extraction region and jointly fit these along with the NUSTAR spectra. The joint spectrum is shown in Figure 8. We only consider data up to $15 \mathrm{keV}$ due to the data being dominated by background above these energies.

We start with a simple power-law model for each source. We do not find evidence for absorption, with upper limits of $2 \times 10^{21} \mathrm{~cm}^{-2}$ and $1 \times 10^{21} \mathrm{~cm}^{-2}$ for the nucleus and extranuclear sources, respectively. We also find an excess of soft X-rays from the extranuclear source that could be due to emission from a photoionized plasma. We fit it with an apec model with the temperature fixed at $0.5 \mathrm{keV}$, in addition to the power-law model. The fit statistic for the joint Chandra plus NuSTAR spectrum with a simple power-law model for each source is $C / \operatorname{DoF}=226.4 / 186$. The fit residuals, shown in Figure 8, show that a simple power-law model does not adequately account for the spectral shape. Allowing a highenergy cutoff for the extranuclear source improves the fit to $C / \mathrm{DoF}=175.5 / 185$. Further allowing the nucleus to have a high-energy cutoff also improves the fit to $164.2 / 184$. For the cutoffpl model of the nucleus, $\Gamma=0.59_{-0.75}^{+0.58}$ and $E_{\mathrm{C}}=3.3_{-1.5}^{+3.6} \mathrm{keV}$.

We list the spectral parameters for both sources in Table 4. The intrinsic $0.5-30 \mathrm{keV}$ X-ray luminosity of the AGN in M51b is $5.4_{-1.0}^{+1.4} \times 10^{38} \mathrm{erg} \mathrm{s}^{-1}$, two orders of magnitude lower than M51a. There is no evidence for an obscured, more powerful AGN in the galaxy from the deep NuSTAR data.

\subsection{The Ultraluminous X-Ray Sources}

The most notable feature in the X-ray spectra of ULXs that is not seen in the X-ray spectra of any sub-Eddington accreting black holes is a spectral turnover below $10 \mathrm{keV}$. This was first seen in high signal-to-noise observations with XMM-Newton (e.g., Stobbart et al. 2006; Gladstone et al. 2009). This spectral shape is generally interpreted to be the superposition of one or more disk-like components. For low signal-to-noise spectra, this continuum shape can be reproduced by a simple phenomenological power law with an exponential cutoff. This is described by $F_{\gamma}=N E^{-\Gamma} e^{-E / E_{C}}$, where $F_{\gamma}$ is the photon flux in units of photons $\mathrm{keV}^{-1} \mathrm{~cm}^{-2} \mathrm{~s}^{-1}$, and $N$ is a constant with the same units. Photon energy is $E$ in $\mathrm{keV}, \Gamma$ is the X-ray spectral index, and $E_{\mathrm{C}}$ is the cutoff energy, also in $\mathrm{keV}$.

Regarding disk models, the standard Shakura \& Sunyaev multicolor thin disk model has been used extensively to model the accretion disk emission from accreting black holes (Shakura \& Sunyaev 1973). This model describes the local temperature of the disk, $T$, as proportional to the radius, $r$, as $T(r) \propto r^{-p}$, where $p=\frac{3}{4}$. However, for high accretion rate systems, a slim disk is expected (Abramowicz et al. 1988). For a slim disk, the local temperature of the disk has a flatter temperature profile as a function of radius with $p \sim \frac{1}{2}$ (Watarai et al. 2000). Slim disks have been proposed as mechanisms to explain ULXs as super-Eddington stellar remnant black hole accretors (e.g., Kato et al. 1998; Poutanen et al. 2007).

In our fits of the joint Chandra and NuSTAR spectra, we use both a cutoff power-law model (cutoffpl in XSPEC) to test for the presence of a spectral turnover and a multicolor disk model with a variable $p$ parameter to test for the emission from a slim disk (diskpbb in XSPEC).

\subsubsection{ULX3 in M51a}

CXOU J132950.6+471155 is located at R.A. $=1329$ 50.68, decl. $=+471155.2$ (J2000) and named ULX3 by Liu \& Mirabel (2005). This ULX is located close enough to the nucleus of M51a that NUSTAR cannot resolve it; therefore, we treat it as part of the spectral fit of the nucleus, as described in Section 3.1. The spectrum of ULX3 can be described well with the cutoff power-law model where $\Gamma=-2.21_{-0.05}^{+0.05}$ and $E_{\mathrm{C}}=$ $1.2_{-0.1}^{+0.2} \mathrm{keV}$. Alternatively, a fit with the diskpbb yields an inner disk temperature of $1.58_{-0.12}^{+0.25} \mathrm{keV}$ and a radial temperature profile index of $p>0.7$. However, the fit statistic is poorer than the cutoffpl model. We show the spectra in Figures 3 and 6. Using the cflux model in XSPEC to calculate the flux of the cutoffpl component, ULX3 has a $0.5-30 \mathrm{keV}$ flux of $2.3_{-0.2}^{+0.3} \times 10^{-13} \mathrm{erg} \mathrm{cm}^{-2} \mathrm{~s}^{-1}$, which, assuming isotropic emission and a distance of $8.5 \mathrm{Mpc}$, implies a luminosity of $2.0_{-0.2}^{+0.3} \times 10^{39} \mathrm{erg} \mathrm{s}^{-1}$.

Previous Chandra and XMM-Newton observations of this source were presented in Terashima \& Wilson (2004) and Dewangan et al. (2005), the authors of which referred to it as source 26 . While there was possible confusion with the nucleus 
Table 3

M51a X-Ray Spectral Parameters

\begin{tabular}{|c|c|c|c|c|c|c|}
\hline $\begin{array}{l}N_{\mathrm{H}} \\
(1)\end{array}$ & $\begin{array}{l}\Gamma \\
(2)\end{array}$ & $\begin{array}{l}f_{\mathrm{C}} \\
(3)\end{array}$ & $\begin{array}{c}\theta_{\mathrm{i}} \\
(4)\end{array}$ & $\begin{array}{c}\chi^{2} / \mathrm{DoF} \\
(5)\end{array}$ & $\begin{array}{l}F_{\mathrm{X}} \\
(6)\end{array}$ & $\begin{array}{l}L_{X} \\
(7)\end{array}$ \\
\hline $\begin{array}{l}\text { Mytorus } \\
24.9_{-0.2}^{+u}, 24.0_{-0.2}^{+0.3}\end{array}$ & $1.58_{-l}^{+0.20}$ & 0.5 & $90.00_{-l}^{+u}, 4.6_{-l}^{+u}$ & $356.9 / 256$ & $2.0_{-0.7}^{+1.1}$ & $1.8_{-0.7}^{+1.0}$ \\
\hline $\begin{array}{l}\text { Borus } \\
25.3_{-0.4}^{+u}\end{array}$ & $<1.43$ & $0.26 \pm 0.03$ & $<22$ & $315.7 / 258$ & $2.0_{-0.6}^{+0.1}$ & $1.7_{-0.5}^{+0.1}$ \\
\hline
\end{tabular}

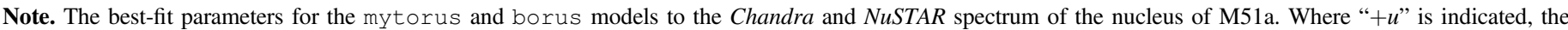

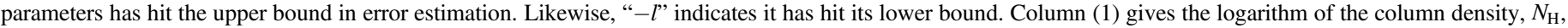

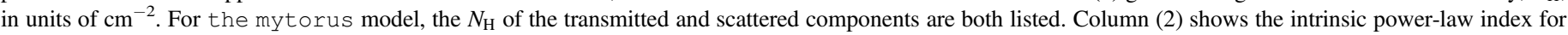

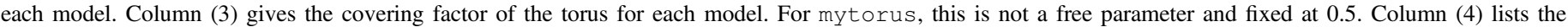

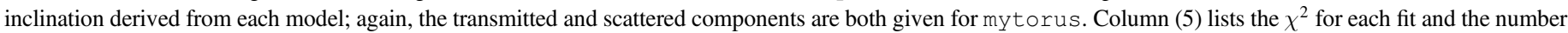

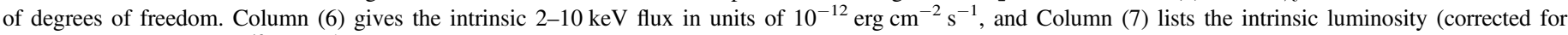
absorption) in units of $10^{40} \mathrm{erg} \mathrm{s}^{-1}$ in that band assuming a distance of $8.58 \mathrm{Mpc}$.

in the XMM-Newton data, both works found the spectrum of the ULX to be consistent with a power-law model, while also noting that the spectrum was very hard. Despite the NuSTAR data also containing the spectrum of the nucleus, our detailed spectral decomposition using Chandra has allowed us to rule out a simple power-law model for this source and has the tightest upper limit on the energy of the cutoff for any ULX studied here.

\subsubsection{ULX5 in M51a}

RX J132954+47145 is located at R.A. = 132953.72 , decl. $=+471435.7(\mathrm{~J} 2000)$ and named ULX5 by Liu \& Mirabel (2005). The $0.5-30 \mathrm{keV}$ spectrum of ULX5 can be described well with a simple power-law model, where $\Gamma=2.23_{-0.31}^{+0.34}$ and with $N_{\mathrm{H}} \sim 4.5 \times 10^{21} \mathrm{~cm}^{-2}$. The fit statistic is $C=179.5$ with 200 DoFs. The inclusion of an exponential cutoff improves the fit to $C=171.7$ with 199 DoFs ( $\Delta C=-7.8$ with the addition of one free parameter).

In order to assess whether the inclusion of this parameter has improved the fit significantly, we run spectral simulations. Using the background and response files for the observed data, we simulate 5000 spectra in XSPEC using the fakeit command based on the best-fit power-law model. We then refit the simulated data in the same way as the observed data, first with the absorbed power-law then with the power-law model with a cutoff, noting the improvement in $C$ each time if any. We find that only in 19 simulated spectra does the addition of a cutoff lead to an improvement in $C$ of 7.8 or more. This represents a false-alarm rate of $0.4 \%$, which is equivalent to a $\sim 3 \sigma$ detection of the cutoff. We therefore conclude that a spectral turnover is present in ULX5.

For the cutoff power-law model, we find $\Gamma=0.89_{-0.81}^{+0.65}$ and $E_{\text {cut }}=3.8_{-1.8}^{+14.6} \mathrm{keV}$. For the diskpbb model, $T_{\text {in }}=$ $2.28_{-0.72}^{+1.83} \mathrm{keV}$ and $p=0.6_{-0.1}^{+0.2}$, with $C=172.9$ with 199 DoFs. We show the spectra in Figure 9 with the data to model ratios for both the power-law and cutoff power-law models.

From the cutoff power-law model, ULX5 has a $0.5-30 \mathrm{keV}$ flux of $1.1_{-0.2}^{+0.3} \times 10^{-13} \mathrm{erg} \mathrm{cm}^{-2} \mathrm{~s}^{-1}$, which, assuming isotropic emission and a distance of $8.5 \mathrm{Mpc}$, implies a luminosity of $9_{-1}^{+3} \times 10^{38} \mathrm{erg} \mathrm{s}^{-1}$.

Results from previous XMM-Newton observations of this source indicated that its spectrum was consistent with a power law, with an added soft component that could be modeled with a multicolor disk or mekal model (Dewangan et al. 2005, their source 41). Winter et al. (2006) also studied this source using
$X M M-N e w t o n$ data, finding that it required a two-component fit, with a blackbody and a power-law component. They measured $\Gamma=1.97$ and a flux of $2.6 \times 10^{-13} \mathrm{erg} \mathrm{cm}^{-2} \mathrm{~s}^{-1}$.

Our sensitivity at low energies is too low to detect this extra component, however. It is also possible that this component is extended and our Chandra data have resolved it out. We check the location around ULX5 in our Chandra data and find a second point source $\sim 5^{\prime \prime}$ to the south. The second source is not bright enough to contribute to the NUSTAR spectrum, with a $3-8 \mathrm{keV}$ count rate $<10 \%$ of ULX5, but it does appear softer than ULX5, which may explain this second component seen in XMM-Newton data.

Our data are the first to show evidence for a spectral turnover at $\sim 10 \mathrm{keV}$ in this source, which has become a hallmark of ULXs.

\subsubsection{ULX7 in M51a}

RX J133001+47137 is located at R.A.+13 30 01.01, decl. $=+471343.9(\mathrm{~J} 2000)$ and was called ULX7 by Liu \& Mirabel (2005). Earnshaw et al. (2016) studied this source in detail, noting its very high short-term variability. They found evidence for a break in the power spectrum similar to that seen in black hole binaries observed in the hard state, suggesting that this ULX is a good IMBH candidate based on these properties.

When fitting the joint Chandra and NuSTAR spectra of this ULX, we found evidence that a cross-calibration constant of unity was not satisfactory, and that a value of $\sim 3$ was required to account for the difference between the Chandra and $N U S T A R$ fluxes. When fitting the spectra between the two NUSTAR observations separately, we found that the source had dropped in flux by a factor of $\sim 4$ from the first observation to the second, over a period of one to two days. Since the Chandra observation overlapped mostly with the first NuSTAR observation, we use only the first observation for spectral analysis.

We then found that the $0.5-30 \mathrm{keV}$ spectrum of ULX7 is fitted well with a simple power-law model with $\Gamma=1.92_{-0.34}^{+0.38}$ and no evidence for absorption above the Galactic column. The fit statistic was $C=110.3$ with 161 DoFs. The inclusion of an exponential cutoff improves the fit statistic to 104.9 for 160 DoFs $(\Delta C=-5.4$ with the addition of one free parameter). Running spectral simulations in the same way as for ULX5, we find that only $41 / 5000$ simulated spectra produce an improvement in $C$ as large as or larger than what we find here. This represents a false-alarm rate of $0.8 \%$, which is equivalent to a 


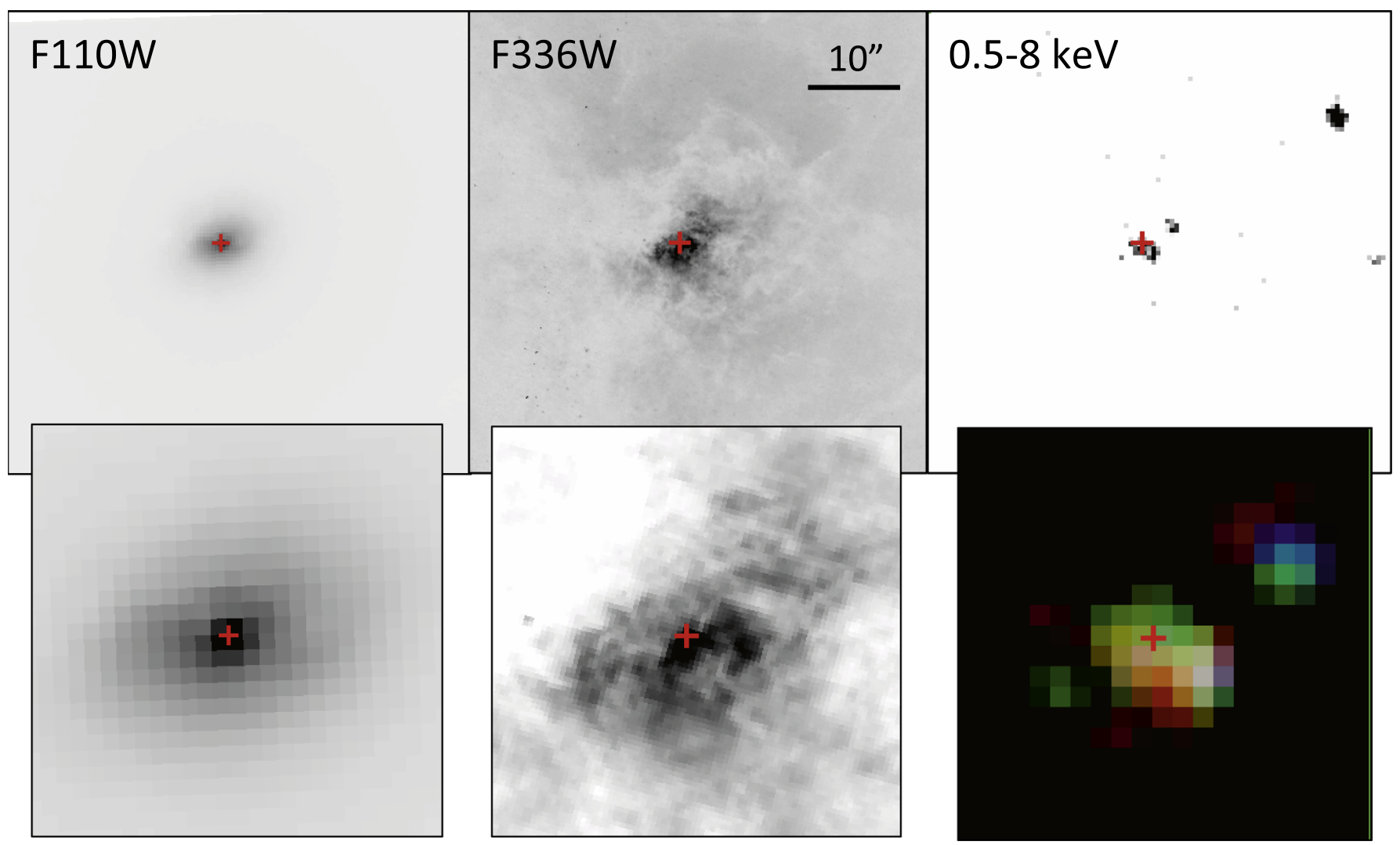

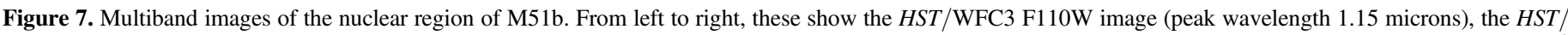

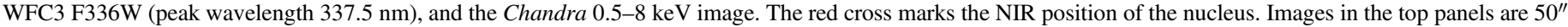

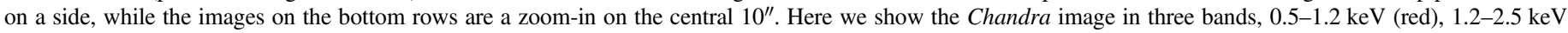
(green), and 2.5-8 keV (blue), which have been smoothed with a Gaussian of $1^{\prime \prime}$.

$\sim 2.6 \sigma$ detection of the cutoff. We therefore conclude that a spectral turnover is present in ULX7.

For the cutoff power-law model, $\Gamma=0.60_{-0.90}^{+1.27}$ and $E_{\mathrm{C}}=$ $3.3_{-1.5}^{+45.6} \mathrm{keV}$. For the diskpbb model, $T_{\text {in }}=2.11_{-0.71}^{+6.46} \mathrm{keV}$ and $p>0.5$, with $C=104.6$ with 160 DoFs. We show the spectra in Figure 10 with the data to model ratios for both the power-law and cutoff power-law models. During the Chandra observation, ULX7 has a $0.5-30 \mathrm{keV}$ flux of $1.2_{-0.2}^{+0.2} \times 10^{-13} \mathrm{erg} \mathrm{cm}^{-2} \mathrm{~s}^{-1}$, which, assuming isotropic emission and a distance of $8.5 \mathrm{Mpc}$, implies a luminosity of $1.2_{-0.2}^{+0.5} \times 10^{39} \mathrm{erg} \mathrm{s}^{-1}$.

Earnshaw et al. (2016) analyzed 5 XMM-Newton and 11 Chandra observations of ULX7, and found that the source exhibits variability of over an order of magnitude in flux from a $0.3-10 \mathrm{keV}$ flux of $\sim 3 \times 10^{-14}-1 \times 10^{-12} \mathrm{erg} \mathrm{cm}^{-2} \mathrm{~s}^{-1}$, but no strong spectral variability. They found a typical $\Gamma \sim 1.5$ when fitting below $10 \mathrm{keV}$ with a simple power-law model. When data from a short NuSTAR observation were included, they showed that inclusion of a cutoff in a power-law spectrum improved their $\chi^{2}$ statistic by 5 , finding $\Gamma=1.3 \pm$ 0.1 and $E_{\mathrm{C}}=18_{-8}^{+43} \mathrm{keV}$. This is broadly consistent with the results we find, within the large uncertainties.

Since Earnshaw et al. (2016) did not find evidence for spectral variability, we investigated using all the NuSTAR data from the new observations rather than just those overlapping with the Chandra data as described above. We accounted for the drop in flux from the source with a variable crosscalibration constant. However, since the source had dropped in flux, using the entire observation, rather than just the segment at high flux, did not improve the counting statistics significantly, and no new constraints could be placed on the parameters, finding $\Gamma=0.69_{-0.90}^{+1.27}$ and $E_{\mathrm{C}}=3.7_{-1.4}^{+u} \mathrm{keV}$ (i.e., unconstrained at the upper end) where $C=185.0$ with 220 DoFs for the cutoffpl model.

\subsubsection{ULX8 in M51a}

RX J133007+47110 is located at R.A. $=133007.55$, decl. $=+471106.1$ (J2000) and was named ULX8 by Liu \& Mirabel (2005) and NGC 5194 X8/ULX5 by Liu \& Bregman (2005). This was recently found to be powered by a neutron-star accretor, implied from the detection of a cyclotron resonance scattering feature (CRSF) in an archival 2012 Chandra observation (Brightman et al. 2018). During the 2012 Chandra observation, the source was observed flaring to fluxes up to $10^{-12} \mathrm{erg} \mathrm{cm}^{-2} \mathrm{~s}^{-1}$, or to luminosities up to $10^{40} \mathrm{erg} \mathrm{s}^{-1}$, from more typical fluxes of $\sim 10^{-13} \mathrm{erg} \mathrm{cm}^{-2} \mathrm{~s}^{-1}$. The CRSF was observed at $4.5 \mathrm{keV}$ with a Gaussian width of $0.1 \mathrm{keV}$ and an equivalent width of $-0.19_{-0.09}^{+0.06} \mathrm{keV}$. Furthermore, the high signal-to-noise Chandra spectrum showed a significant departure from a simple power-law spectrum. The continuum could be fitted by a power-law model $(\Gamma=1.3 \pm 0.3)$ with an exponential cutoff $\left(E_{\mathrm{C}}=3.7_{-1.0}^{+2.2} \mathrm{keV}\right)$.

During our new Chandra and NUSTAR observations, the source was observed at a much lower $0.5-30 \mathrm{keV}$ flux of $1.6_{-0.2}^{+0.2} \times 10^{-13} \mathrm{erg} \mathrm{cm}^{-2} \mathrm{~s}^{-1}$. The $0.5-30 \mathrm{keV}$ spectrum can be described well with a simple power-law model with $\Gamma=1.94_{-0.20}^{+0.23}$, where $C=228.7$ with 304 degrees of freedom. The inclusion of an exponential cutoff does not improve the fit 

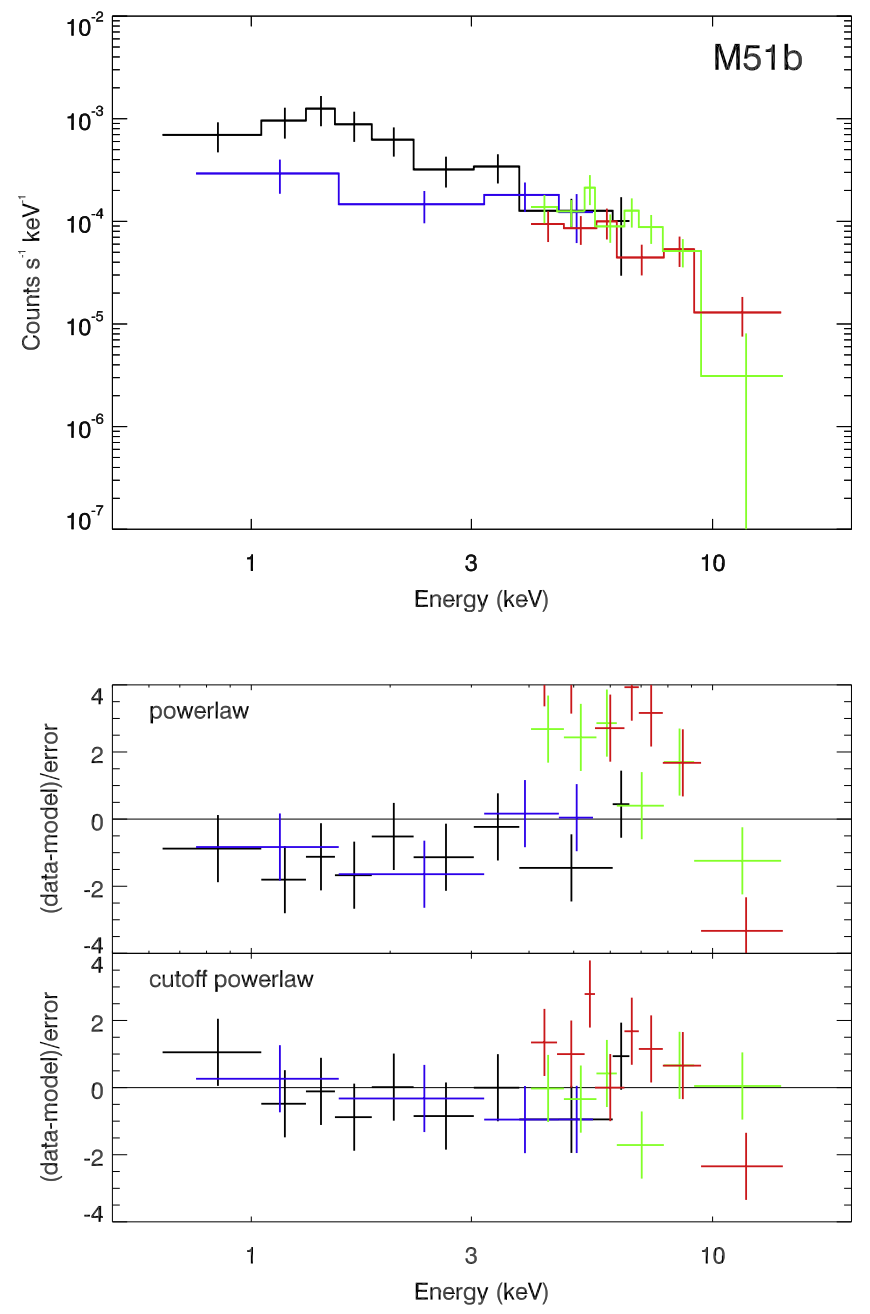

Figure 8. Chandra (the nucleus in black and the extranuclear source in blue) and NuSTAR (FPMA in red and FPMB in green) spectra of the M51b nuclear region (top panel). Residuals to a fit with a power-law model for both sources and a cutoff power-law model for both are also shown (bottom panels).

Table 4

M51b X-Ray Spectral Parameters

\begin{tabular}{lcccc}
\hline \hline$\Gamma$ & $E_{\mathrm{C}}$ & $C / \mathrm{DoF}$ & $F_{\mathrm{X}}$ & $L_{\mathrm{X}}$ \\
$(1)$ & $(2)$ & $(3)$ & $(4)$ & $(5)$ \\
\hline $\begin{array}{l}\text { Nucleus } \\
0.59_{-0.75}^{+0.58}\end{array}$ & $3.3_{-1.5}^{+3.6}$ & $164.2 / 184$ & $6.2_{-1.2}^{+1.7}$ & $5.4_{-1.0}^{+1.4}$ \\
$\begin{array}{l}\text { Extranuclear source } \\
<1.6\end{array}$ & & & \\
\hline
\end{tabular}

Note. The best-fit parameters for the cutoff power-law model to the Chandra and NuSTAR spectrum of the nuclear sources of M51b. Column (1) gives the power-law index of the model, and column (2) lists the cutoff energy of the cutoff power-law model in $\mathrm{keV}$. Column (3) gives the $C$-statistic of the fit and the number of degrees of freedom, column (4) gives the unabsorbed flux in the range $0.5-30 \mathrm{keV}$ in units of $10^{-14} \mathrm{erg} \mathrm{cm}^{-2} \mathrm{~s}^{-1}$, and column (5) gives the luminosity assuming a distance of $8.58 \mathrm{Mpc}$ to M51 in units of $10^{38} \mathrm{erg} \mathrm{s}^{-1}$.

statistic significantly $(\Delta C=-0.2$ with the addition of one free parameter), and the parameters of the diskpbo model are not constrained by the data. We show the spectra in Figure 11 with the data to model ratios for both the power-law and cutoff power-law models.
We furthermore find no evidence for absorption lines in the new data. Adding an absorption line at $4.5 \mathrm{keV}$ does not improve the fit statistic. However, since our new observations found this source at a lower flux than the 2012 Chandra observation, the CRSF is not likely to be detectable. The $90 \%$ confidence lower limit on the equivalent width of an absorption line at $4.5 \mathrm{keV}$ is $-0.18 \mathrm{keV}$, which is consistent with that measured in the 2012 Chandra data. The shape of the X-ray spectrum measured here is marginally consistent with that measured in the 2012 Chandra data, but we cannot rule out spectral evolution with flux.

Dewangan et al. (2005) also found that this source (their source \#82) was consistent with an absorbed power law from its XMM-Newton spectrum, with $\Gamma=2.4 \pm 0.2$ observed at a slightly higher flux of $2.6 \times 10^{-13} \mathrm{erg} \mathrm{cm}^{-2} \mathrm{~s}^{-1}$. They also found higher absorption than evident in our Chandra spectrum with $N_{\mathrm{H}}=1.6 \pm 0.4 \times 10^{21} \mathrm{~cm}^{-2}$. Yoshida et al. (2010) analyzed three Chandra and four XMM-Newton observations of this source, again finding that its spectrum is consistent with a power law.

\subsubsection{ULX9 in M51b}

RX J133006+47156 is located at R.A.+13 30 06.00, decl. $=+471542.3$ (J2000) and was called ULX9 by Liu \& Mirabel (2005). A fit with an absorbed power-law model to the $0.5-30 \mathrm{keV}$ spectrum of ULX9 gives $\Gamma=2.34_{-0.30}^{+0.33}$ and $N_{\mathrm{H}} \sim 7.8 \times 10^{21} \mathrm{~cm}^{-2}$. The fit statistic is $C=271.5$ with 243 degrees of freedom. Including an exponential cutoff improves the fit to $C=258.1$ with 242 degrees of freedom $(\Delta C=-13.4$ with the addition of one free parameter). Spectral simulations show that only $1 / 5000$ simulated spectra produce an improvement in $C$ as large as or larger than what we find here. This represents a false-alarm rate of $\sim 0.02 \%$, which is equivalent to a $>3 \sigma$ detection of the cutoff. We therefore conclude that a spectral turnover is present in ULX9.

We find $\Gamma=0.35_{-1.05}^{+1.26}$ and $E_{\mathrm{C}}=2.3_{-1.3}^{+4.2} \mathrm{keV}$ for the cutoffpl model. For the diskpbb model, $T_{\text {in }}=$ $1.82_{-0.47}^{+1.21} \mathrm{keV}$ and $p$ is unconstrained. Here, $C=257.7$ with 242 DoFs. From the cutoff power-law model ULX9 has a $0.5-30 \mathrm{keV}$ flux of $9_{-1}^{+2} \times 10^{-14} \mathrm{erg} \mathrm{cm}^{-2} \mathrm{~s}^{-1}$, which, assuming isotropic emission and a distance of $8.5 \mathrm{Mpc}$, implies a luminosity of $8_{-1}^{+2} \times 10^{38} \mathrm{erg} \mathrm{s}^{-1}$. We show the spectra in Figure 12 with the data to model ratios for both the powerlaw and cutoff power-law models.

Previous modeling of the XMM-Newton spectrum found that a power law alone could explain the shape (Dewangan et al. 2005), but at a higher flux of $1.2 \times 10^{-13} \mathrm{erg} \mathrm{cm}^{-2} \mathrm{~s}^{-1}$.

\subsection{6. $J 132946+471041$}

RX J132946+47107 is located at R.A. $=1329$ 46.11, decl. $=+471042.3(\mathrm{~J} 2000)$. The $0.5-30 \mathrm{keV}$ spectrum of this source is described well by a power-law model with $\Gamma=1.64_{-0.16}^{+0.26}$, no absorption above the Galactic $N_{\mathrm{H}}$, and no evidence for a cutoff. The parameters of the diskpbb model are unconstrained. The $0.5-30 \mathrm{keV}$ flux is $1.1 \times 10^{-13}$ $\mathrm{erg} \mathrm{cm} \mathrm{cm}^{-2} \mathrm{~s}^{-1}$, implying a luminosity of $9_{-3}^{+2} \times 10^{38} \mathrm{erg} \mathrm{s}^{-1}$. We show the spectra in Figure 13 with the data to model ratios for both the power-law and cutoff power-law models. 
Table 5

Extranuclear Point Source X-Ray Spectral Parameters

\begin{tabular}{|c|c|c|c|c|c|c|}
\hline $\begin{array}{l}\text { Name and model } \\
\text { (1) }\end{array}$ & $\begin{array}{l}N_{\mathrm{H}} \\
(2)\end{array}$ & $\begin{array}{c}\Gamma \text { or } T_{\text {in }} \\
(3)\end{array}$ & $\begin{array}{c}E_{\mathrm{C}} \text { or } p \\
\text { (4) }\end{array}$ & $\begin{array}{l}C / \mathrm{DoF} \\
\text { (5) }\end{array}$ & $\begin{array}{l}F_{X} \\
(6)\end{array}$ & $\begin{array}{l}L_{X} \\
(7)\end{array}$ \\
\hline \multicolumn{7}{|l|}{ ULX3 } \\
\hline Cutoffpl & 0.0 & $-2.21_{-0.05}^{+0.05}$ & $1.2_{-0.1}^{+0.2}$ & $315.7 / 258$ & $2.3_{-0.2}^{+0.3}$ & $2.0_{-0.2}^{+0.3}$ \\
\hline \multicolumn{7}{|l|}{ ULX5 } \\
\hline Powerlaw & $4.5_{-3.6}^{+4.2}$ & $2.23_{-0.31}^{+0.34}$ & $\cdots$ & $179.5 / 200$ & $1.5_{-0.2}^{+0.3}$ & $1.3_{-0.2}^{+0.3}$ \\
\hline Cutoffpl & $<4.7$ & $0.89_{-0.81}^{+0.65}$ & $3.8_{-1.8}^{+14.6}$ & $171.7 / 199$ & $1.1_{-0.2}^{+0.3}$ & $0.9_{-0.1}^{+0.3}$ \\
\hline \multicolumn{7}{|l|}{ ULX7 } \\
\hline Powerlaw & $<3.2$ & $1.92_{-0.34}^{+0.38}$ & $\ldots$ & $110.3 / 161$ & $1.8_{-0.3}^{+0.5}$ & $1.6_{-0.3}^{+0.4}$ \\
\hline Cutoffpl & $<3.1$ & $0.60_{-0.90}^{+1.27}$ & $3.3_{-1.5}^{+45.6}$ & $104.9 / 160$ & $1.2_{-0.2}^{+0.2}$ & $1.2_{-0.2}^{+0.5}$ \\
\hline Diskpbb & $<4.5$ & $2.11_{-0.71}^{+6.46}$ & $>0.5$ & $104.6 / 160$ & $1.4_{-0.2}^{+0.5}$ & $1.2_{-0.2}^{+0.4}$ \\
\hline \multicolumn{7}{|l|}{ ULX8 } \\
\hline Powerlaw & $<0.9$ & $1.94_{-0.20}^{+0.23}$ & $\ldots$ & $228.7 / 304$ & $1.6_{-0.2}^{+0.2}$ & $1.4_{-0.2}^{+0.2}$ \\
\hline Cutoffpl & $<1.5$ & $0.35_{-1.06}^{+1.26}$ & $2.3_{-1.3}^{+4.2}$ & $258.1 / 242$ & $0.9_{-0.1}^{+0.2}$ & $0.8_{-0.1}^{+0.2}$ \\
\hline Diskpbb & $<2.3$ & $1.82_{-0.47}^{+1.21}$ & $0.7_{-l}^{+u}$ & $257.7 / 242$ & $0.9_{-0.1}^{+0.2}$ & $0.8_{-0.1}^{+0.2}$ \\
\hline \multicolumn{7}{|l|}{$132946+471041$} \\
\hline Powerlaw & $<0.1$ & $1.64_{-0.16}^{+0.26}$ & $\ldots$ & $221.8 / 303$ & $1.1_{-0.2}^{+0.2}$ & $0.9_{-0.2}^{+0.2}$ \\
\hline Cutoffpl & $<0.1$ & $1.62_{-0.43}^{+0.26}$ & $500.0_{-l}^{+u}$ & $219.5 / 223$ & $1.1_{-0.3}^{+0.2}$ & $0.9_{-0.3}^{+0.2}$ \\
\hline Diskpbb & $<2.7$ & $10.00_{-5.48}^{+u}$ & $0.6_{-l}^{+u}$ & $220.4 / 223$ & $1.0_{-0.2}^{+0.2}$ & $0.8_{-0.2}^{+0.2}$ \\
\hline \multicolumn{7}{|l|}{$132959+471052$} \\
\hline Powerlaw & $<3.0$ & $1.33_{-0.21}^{+0.28}$ & $\ldots$ & $198.8 / 177$ & $0.8_{-0.2}^{+0.3}$ & $0.7_{-0.2}^{+0.3}$ \\
\hline Cutoffpl & 0.0 & $1.13_{-0.66}^{+0.34}$ & $23.8_{-l}^{+u}$ & $198.2 / 176$ & $0.6_{-0.1}^{+0.2}$ & $0.5_{-0.1}^{+0.1}$ \\
\hline Diskpbb & $<2.6$ & $7.98_{-4.67}^{+u}$ & $0.6_{-0.1}^{+0.1}$ & $198.2 / 176$ & $0.6_{-0.1}^{+0.2}$ & $0.5_{-0.1}^{+0.1}$ \\
\hline
\end{tabular}

Note. Spectral parameters of the power-law, cutoff power-law, and diskpbb model fits to the Chandra and NuSTAR spectra of the extranuclear point sources. For ULX3, this is part of the data set on the nucleus. Column (2) gives the absorption column intrinsic to the source in units of $10^{21} \mathrm{~cm}^{-2}$. " 0.0 " indicates that no absorption was detected on top of the Galactic absorption. Column (3) gives the power-law index of the power-law and cutoff power-law models or the temperature of the diskpbb model in $\mathrm{keV}$, and column (4) lists the cutoff energy of the cutoff power-law model in keV or the index of the radial temperature profile of the diskpbb model. "u" and/or "l" indicate that the parameter was unconstrained at the upper or lower end. Column (5) gives the $C$-statistic of the fit and the number of degrees of freedom, column (6) gives the unabsorbed flux in the range of $0.5-30 \mathrm{keV}$ in units of $10^{-13} \mathrm{erg} \mathrm{cm}^{-2} \mathrm{~s}^{-1}$, and column (7) gives the luminosity assuming a distance of $8.58 \mathrm{Mpc}$ to M51 in units of $10^{39} \mathrm{erg} \mathrm{s}^{-1}$.

\subsection{7. $J 132959+471052$}

CXOU J132957.5+471048 is located at R.A. $=1329$ 57.57 , decl. $=+471048.3(\mathrm{~J} 2000)$ and was called XMM6 by Winter et al. (2006). The $0.5-30 \mathrm{keV}$ spectrum of this source is described well by a power-law model with $\Gamma=1.33_{-0.21}^{+0.28}$, no absorption above the Galactic $N_{\mathrm{H}}$, and no evidence for a cutoff. The $0.5-30 \mathrm{keV}$ flux is $7.6 \times 10^{-14} \mathrm{erg} \mathrm{cm}^{-2} \mathrm{~s}^{-1}$ implying a luminosity of $7 \times 10^{38} \mathrm{erg} \mathrm{s}^{-1}$. We show the spectra in Figure 14 with the data to model ratios for both the powerlaw and cutoff power-law models.

\section{Discussion}

\subsection{The Dual Active Galactic Nuclei}

Dual AGNs occur when a pair of galaxies separated on kiloparsec scales are simultaneously both observed to host an AGN (e.g., NGC 6240, Komossa et al. 2003) and are predicted by merger-driven AGN models to occur. The dual AGN of M51 are only the second to be resolved above $10 \mathrm{keV}$ with $N U S T A R$ after MCG +04-48-002 and NGC 6921 (Koss et al. 2016). They were only recently detected by Swift/BAT, but their individual hard
X-ray emission could not be resolved (Oh et al. 2018). Prior to NuSTAR and Swift/BAT, the nucleus of M51a has been studied at hard X-ray wavelengths most notably by Fukazawa et al. (2001), where a BeppoSAX observation of the galaxy was reported. The authors inferred a column density of $\sim 10^{24} \mathrm{~cm}^{-2}$ for the nucleus based on the excess of hard emission over that seen at softer energies. An analysis of a more recent $\sim 20 \mathrm{ks} N U S T A R$ observation of M51 in conjunction with deep archival Chandra data was presented in $\mathrm{Xu}$ et al. (2016), where the intrinsic $L_{\mathrm{X}}, \Gamma$, and $N_{\mathrm{H}}$ were estimated. However, the low signal to noise of the NUSTAR data did not allow measurement of the torus covering factor, which requires good quality data above $10 \mathrm{keV}$. Furthermore, the NuSTAR observation lacked the simultaneous Chandra data we obtained to resolve out the variable extranuclear emission. Xu et al. (2016) inferred $L_{\mathrm{X}}=4 \times 10^{40} \mathrm{erg} \mathrm{s}^{-1}, \Gamma=1.8 \pm 0.3$, and $N_{\mathrm{H}}=$ $7 \pm 3 \times 10^{24} \mathrm{~cm}^{-2}$ with the mytorus model. For that model, we obtain $L_{\mathrm{X}}=1.8_{-0.7}^{+1.0} \times 10^{40} \mathrm{erg} \mathrm{s}^{-1}, \Gamma=1.58_{-l}^{+0.20}$, and $N_{\mathrm{H}}=$ $7.1_{-2.4}^{+u} \times 10^{24} \mathrm{~cm}^{-2}$. The $N_{\mathrm{H}}$ values are in good agreement, but our luminosity estimate is slightly lower, and our estimate of the intrinsic photon index is harder.

Gandhi et al. (2009) noted that there exists a very tight relationship between the intrinsic X-ray luminosity and the 12 micron 


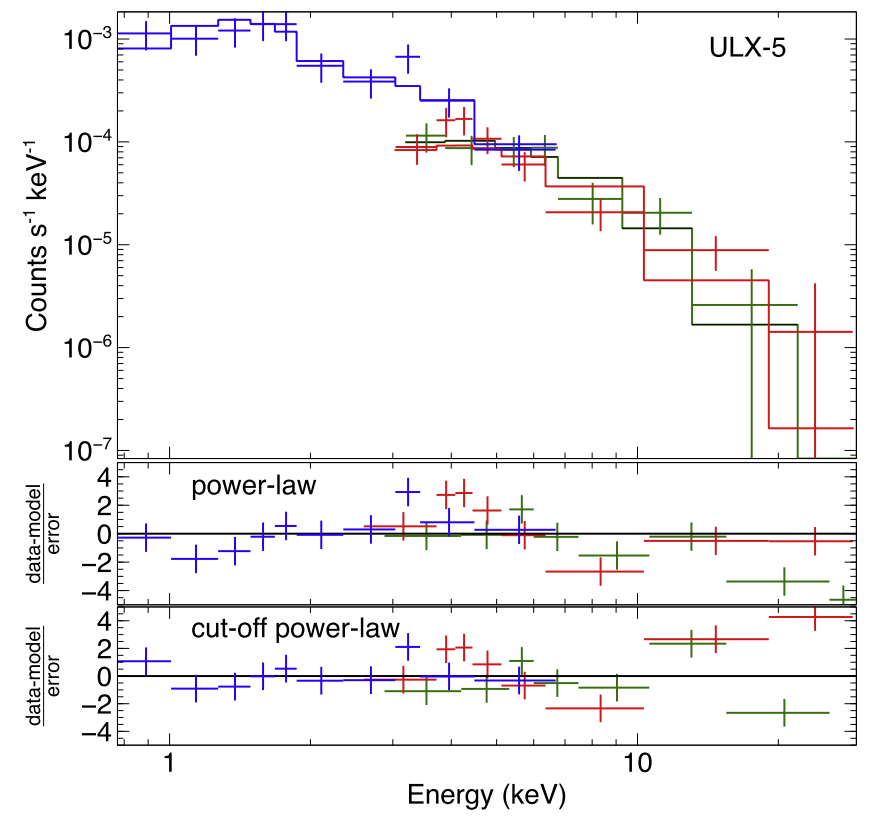

Figure 9. Chandra (blue) and NUSTAR (FPMA in red and FPMB in green) spectra of ULX5 fitted with the cutoff power-law model are shown in the top panel. Residuals to the power-law and cutoff power-law models are shown in the bottom panels.

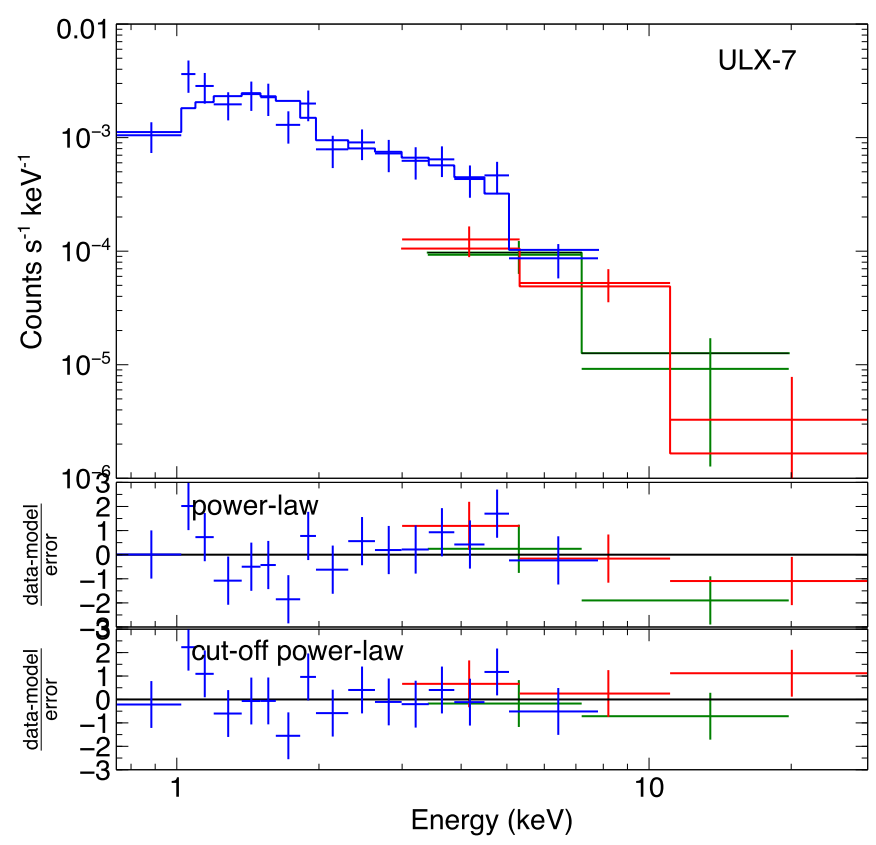

Figure 10. Chandra (blue) and NuSTAR (FPMA in red and FPMB in green) spectra of ULX7 fitted with the cutoff power-law model are shown in the top panel. Residuals to the power-law and cutoff power-law models are shown in the bottom panels.

luminosity of the AGN where the nucleus has been resolved in the mid-infrared and a good estimate of the intrinsic X-ray luminosity exists. From the latest relationship published by Asmus et al. (2015), given an intrinsic X-ray luminosity of $2 \times 10^{40} \mathrm{erg} \mathrm{s}^{-1}$, the predicted 12 micron luminosity is $5 \times 10^{40} \mathrm{erg} \mathrm{s}^{-1}$. This is exactly the value observed for M51a from high-resolution MIR imaging and thus adds support to this relationship down to the lowest observed luminosities of $\sim 10^{40} \mathrm{erg} \mathrm{s}^{-1}$.

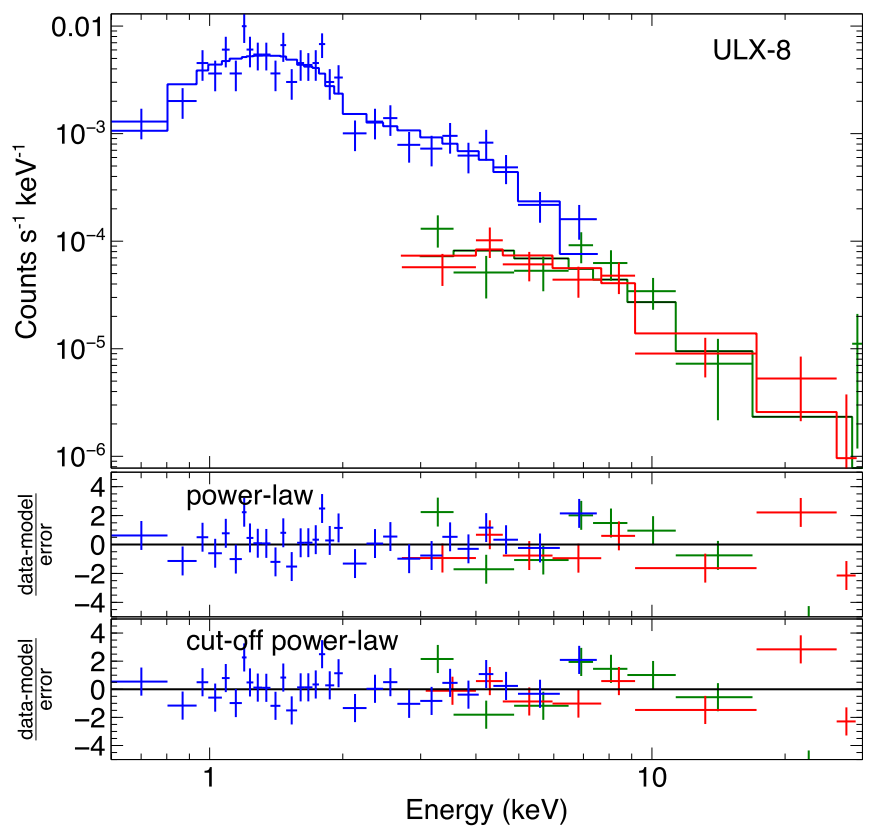

Figure 11. Chandra (blue) and NuSTAR (FPMA in red and FPMB in green) spectra of ULX8 fitted with the power-law model are shown in the top panel. Residuals to the power-law and cutoff power-law models are shown in the bottom panels.

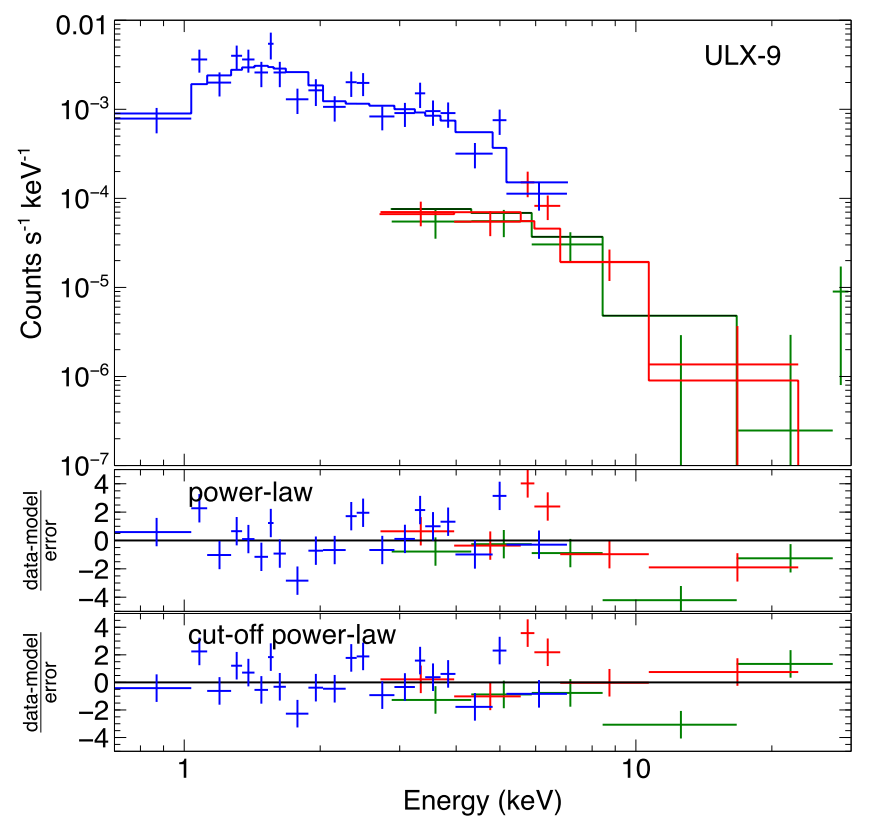

Figure 12. Chandra (blue) and NUSTAR (FPMA in red and FPMB in green) spectra of ULX9 fitted with the cutoff power-law model are shown in the top panel. Residuals to the power-law and cutoff power-law models are shown in the bottom panels.

We calculated the black hole mass of M51a using the $M_{\mathrm{BH}}-\sigma_{*}$ relation from Kormendy \& Ho (2013), where $\log \left(M_{\mathrm{BH}} / M_{\odot}\right)=$ $4.38 \times \log \left(\sigma_{*} / 200 \mathrm{~km} \mathrm{~s}^{-1}\right)+8.49$. We used the $\sigma_{*}$ inferred from the Ca II triplet measurement of $63 \pm 4 \mathrm{~km} \mathrm{~s}^{-1}$ to determine a black hole mass as the $3950 \AA$ to $5500 \AA$ region velocity dispersion was close to the instrumental resolution. This yielded $\log \left(M_{\mathrm{BH}} / M_{\odot}\right)=6.3 \pm 0.4$, where the uncertainty has been propagated from the measurement uncertainty on the stellar dispersion, which is larger than the intrinsic scatter of the 


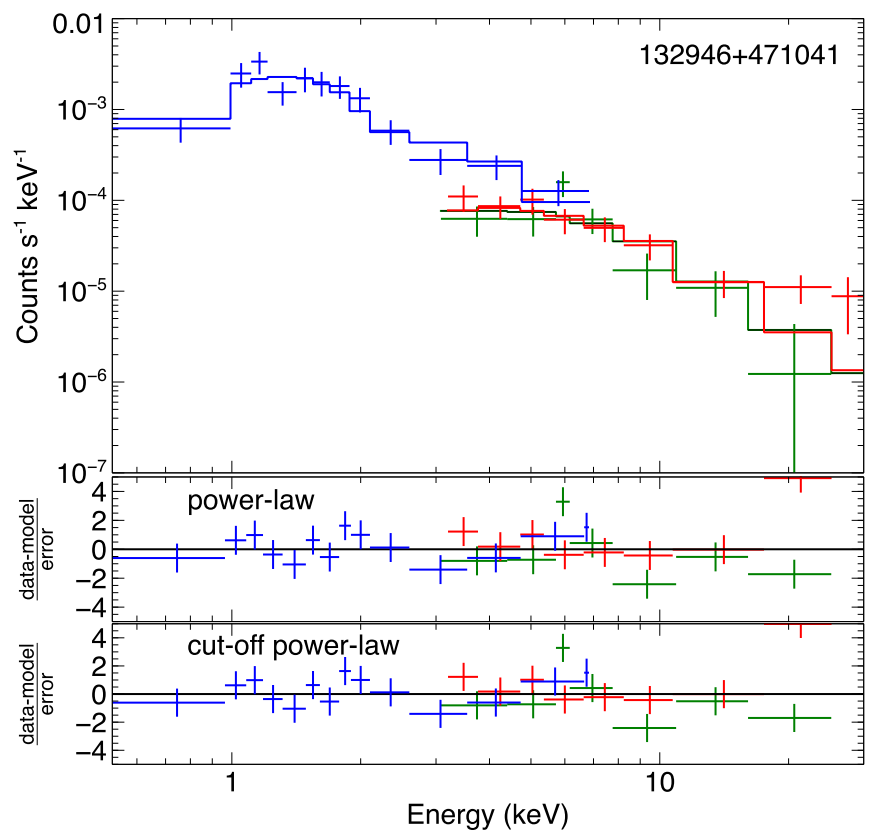

Figure 13. Chandra (blue) and NUSTAR (FPMA in red and FPMB in green) spectra of J132946+471041 fitted with the power-law model are shown in the top panel. Residuals to the power-law and cutoff power-law models are shown in the bottom panels.

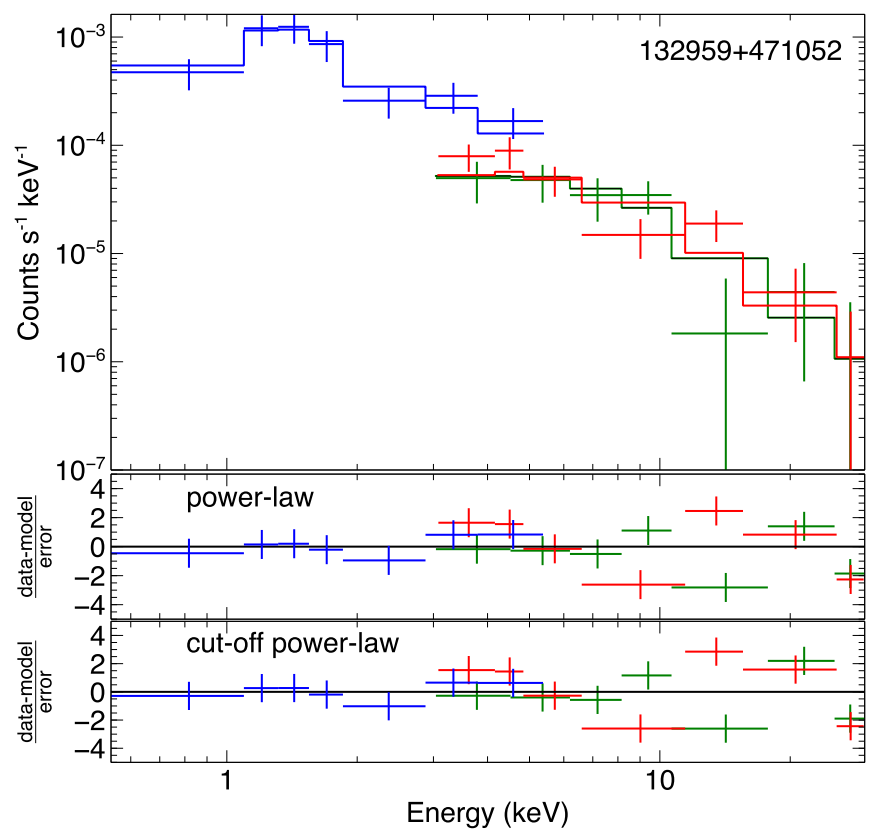

Figure 14. Chandra (blue) and NuSTAR (FPMA in red and FPMB in green) spectra of J132959+471052 fitted with the power-law model are shown in the top panel. Residuals to the power-law and cutoff power-law models are shown in the bottom panels.

relationship of 0.29 (Kormendy \& Ho 2013). The mass of the black hole in the nucleus of M51a was previously estimated to be $\log \left(M_{\mathrm{BH}} / M_{\odot}\right)=6.95$ by Woo \& Urry (2002), where a stellar dispersion value of $102 \mathrm{~km} \mathrm{~s}^{-1}$ from Nelson \& Whittle (1995) was used. Our stellar dispersion measurement is smaller due to the better spectral resolution of our measurement. We note that Ho et al. (2009) also measured a lower velocity dispersion of $76.3 \pm 9.1 \mathrm{~km} \mathrm{~s}^{-1}$ for M51a, also at Palomar.

We calculate the bolometric luminosity, and subsequently the Eddington ratio of M51a, by applying a bolometric correction, $\kappa_{\mathrm{Bol}}$, to the X-ray luminosity. The X-ray luminosity of $\sim 10^{40} \mathrm{erg} \mathrm{s}^{-1}$ is lower than studies of $\kappa_{\mathrm{Bol}}$ have used (e.g., Marconi et al. 2004; Lusso et al. 2012). These have found a decreasing trend of $\kappa_{\mathrm{Bol}}$ with luminosity, with $\kappa_{\mathrm{Bol}}=10$ shown to be appropriate for low-luminosity AGNs, including Compton-thick ones (Brightman et al. 2017). Using $\kappa_{\mathrm{Bol}}=10$ implies M51a has a bolometric luminosity of $\sim 10^{41} \mathrm{erg} \mathrm{s}^{-1}$ and an Eddington ratio of $\lambda_{\text {Edd }} \sim 10^{-4}$.

The low measured value of the photon index of 1.4-1.8 (depending on the model used) for M51a is consistent with a low Eddington rate system (e.g., Shemmer et al. 2006; Brightman et al. 2013; Trakhtenbrot et al. 2017), even when modeling the spectra of Compton-thick AGNs with torus models (Brightman et al. 2016). For $\lambda_{\text {Edd }} \sim 10^{-4}$, the $\Gamma-\lambda_{\text {Edd }}$ relationship predicts a range in $\Gamma$ of $1.2-1.5$ from the fit to the full BASS sample presented in Trakhtenbrot et al. (2017), and therefore, our results are fully consistent with this relationship.

The luminosity and Eddington ratio regime of M51a is an extremely low one, being the lowest luminosity Compton-thick AGN known, slightly less luminous than the recently identified low-luminosity CTAGN in NGC 1448 (Annuar et al. 2017). This allows us to test various models of torus formation in a regime where the torus is predicted to disappear or be diminished. Based on a model where the torus is produced by outflowing material, Elitzur \& Shlosman (2006) suggested that the obscuring torus disappears below a bolometric luminosity of $10^{42} \mathrm{erg} \mathrm{s}^{-1}$. However, the mere detection of a Compton-thick line of sight to the AGN shows that this is not the case. Furthermore, the torus covering factor for the AGN in M51a was inferred to be $0.26_{-0.02}^{+0.02}$ from the borus model, showing that while the torus subtends a small fraction of the sky, it is a significant one. The radiation-driven fountain model of Wada (2015) also predicts a diminished torus at low luminosities, or more specifically, Eddington ratios; however, their model still predicts a covering factor of $0.1-0.3$ at the low end of their $L_{X}$ range, having peaked at higher $L_{X}$, around $10^{43}-10^{44} \mathrm{erg} \mathrm{s}^{-1}$. Their calculations do not consider X-ray luminosities as low as that observed from M51a.

On the other hand, the fact that M51a is in an ongoing merger with M51b may be more pertinent regarding the source of the obscuration, since gas will have been driven into the nucleus during this merger process. Indeed, recent results on mergers show that AGNs in these systems show increased incidence of Compton-thick obscuration with respect to those not in mergers (Kocevski et al. 2015; Ricci et al. 2017a). This may explain the presence of a large amount of obscuring material in the nucleus of M51a despite its low accretion rate and luminosity. The fact that a prominent reflection component is observed in the X-ray spectrum of M51a suggests that the obscuration is being carried out by a torus-like structure. This is because the X-ray source needs to be both reflected by Compton-thick material out of the line of sight and obscured by material in the line of sight to produce such a feature. Chandra resolves this emission at $\sim 1^{\prime \prime}(\sim 40 \mathrm{pc}$ at $8.58 \mathrm{Mpc})$ scales, confirming that the obscuring material is very close to the nucleus and not on galactic scales. The fact that the X-ray and MIR luminosities of M51a lie on the same relationship as other local AGN also implies that this material is on the same scales as the obscuring torus.

We compare the covering factor that we have derived to the local AGN obscured fraction as a function of X-ray luminosity, 


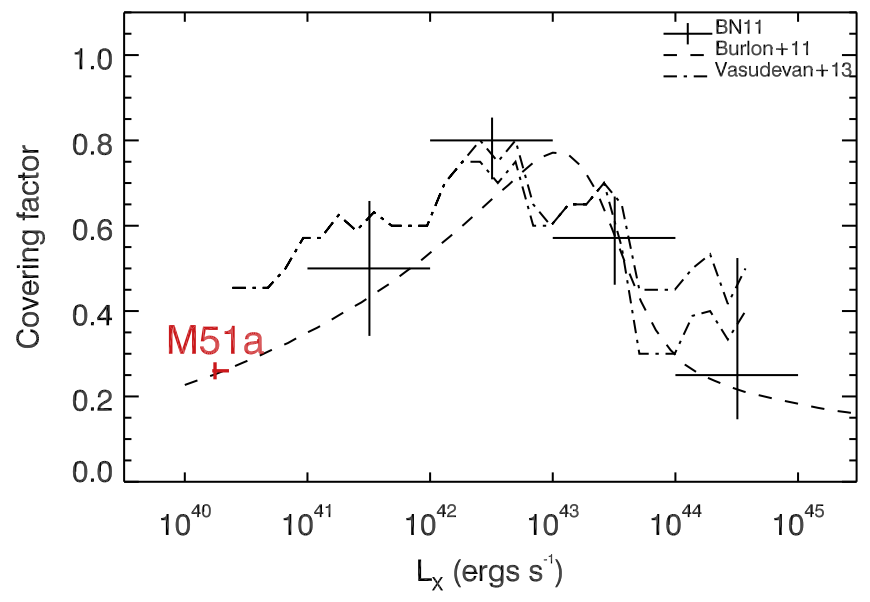

Figure 15. Covering factor of the torus in the AGN of M51a derived from the borus model, plotted in red. We compare this covering factor to the local AGN obscured fraction as derived by Burlon et al. (2011), Brightman \& Nandra (2011b), and Vasudevan et al. (2013).

which is the average torus covering factor, as derived by Burlon et al. (2011), Brightman \& Nandra (2011b), and Vasudevan et al. (2013), and show the comparison in Figure 15. The results agree very well, showing that M51a supports the decline in the torus covering factor at low X-ray luminosities. Previous results on inferring the covering factor of the torus in Compton-thick AGNs at higher luminosities have also shown good agreement with the obscured fraction (e.g., Brightman et al. 2015). The luminosity dependence of the obscured fraction, which has often been tied to the increasing dust sublimation radius with luminosity, has more recently been attributed to an accretion rate dependence (e.g., Ricci et al. 2017b). Ricci et al. (2017b) found from a large sample of local Swift/BAT-detected AGNs that the obscured fraction shows a sharp drop above $\lambda_{\text {Edd }} \sim 0.01$, which corresponds to the effective Eddington limit of dusty gas.

While it was previously concluded that the torus in M51a must have a large covering factor in order to account for the high $\mathrm{EW}$ of its Fe K $\alpha$ line (Levenson et al. 2002), our new data combined with the latest borus model find that the covering factor is relatively low. The latest calculations presented in Baloković et al. (2018) show that the high EW of $3.3 \mathrm{keV}$ can be produced even for low covering factors, especially when the line-of-sight $N_{\mathrm{H}}$ is high, as it is for M51a, and also dependent on viewing perspective. The high $\mathrm{EW}$ of the $\mathrm{Fe} \mathrm{K} \alpha$ line given the low luminosity of M51a is also consistent with the X-ray Baldwin effect, otherwise known as the Iwasawa-Taniguchi effect (Iwasawa \& Taniguchi 1993), which describes an anticorrelation between the $\mathrm{Fe} \mathrm{K} \alpha \mathrm{EW}$ and the intrinsic $\mathrm{X}$-ray luminosity. This was recently explored for a sample of CTAGNs in Boorman et al. (2018), finding that the relationship holds even for these sources and may be due to the luminositydependent torus covering factor (e.g., Ricci et al. 2013).

For both the borus and mytorus models, we found that allowing the scattered and transmitted components to be decoupled from one another leads to an improvement in the fit statistic, implying that the smooth toroidal geometries that these models describe are too simplistic. This is likely due to the fact that the torus is clumpy, rather than smooth, as described by the simplest unified scheme.

Several works have estimated the intrinsic X-ray luminosity of the AGN in M51b (e.g., Hernández-García et al. 2014); however, due to its low luminosity, several bright non-nuclear sources have made this challenging, even for Chandra observations where M51b was off-axis. With our latest observation, where M51b was closer to the optical axis than previous observations, we have resolved the nuclear region, finding that its true luminosity is even lower than previous estimates with $L_{\mathrm{X}}=5 \times 10^{38} \mathrm{erg} \mathrm{s}^{-1}$. This was similarly found by Rampadarath et al. (2018) using the same Chandra data that we use. A. Annuar et al. (2018, in preparation) also analyzed these data as part of an investigation into the $N_{\mathrm{H}}$ distribution of AGNs within $15 \mathrm{Mpc}$. Their $L_{\mathrm{X}}$ estimate for M51b is also in agreement with ours.

We calculated the black hole mass of M51b in the same way as M51a as above using $\sigma_{*}=124.8 \pm 8.1 \mathrm{~km} \mathrm{~s}^{-1}$ from Ho et al. (2009). This yielded $\log \left(M_{\mathrm{BH}} / M_{\odot}\right)=7.6 \pm 0.5$. This is consistent with the value calculated by Schlegel et al. (2016) of 7.6. It may be surprising that the mass of the SMBH in M51b is more massive than that in M51a since M51b is often named a dwarf galaxy. However, M51b has a total stellar mass of $2.5 \times 10^{10} M_{\odot}$, which is more than half the stellar mass of M51a with $4.7 \times 10^{10} M_{\odot}$ (Mentuch Cooper et al. 2012). Both galaxies are consistent with the distribution of $M_{\mathrm{BH}}$ and $M_{*}$ presented in e.g., Reines \& Volonteri (2015). M51b nevertheless has a higher $M_{\mathrm{BH}} / M_{*}$ ratio than the average and M51a has a lower one.

Given its X-ray luminosity and a bolometric correction of 10 implies the AGN in M51b has a bolometric luminosity of $5 \times 10^{39} \mathrm{erg} \mathrm{s}^{-1}$ and therefore an Eddington ratio of $\lambda_{\text {Edd }} \sim 10^{-6}$. M51b also has a detection by Spitzer/IRS of the mid-IR line [Ne V] at $14.3 \mu \mathrm{m}$, which, due to its high ionization potential, is considered a good tracer of AGN activity (Goulding \& Alexander 2009). Gruppioni et al. (2016) calibrated a relationship between $L_{\mathrm{Bol}}$, derived from IR torus modeling, and the [Ne V] line luminosity. For our derived $L_{\mathrm{Bol}}$ value, the implied [Ne V] line luminosity is $2.8 \times 10^{37} \mathrm{erg} \mathrm{s}^{-1}$. The observed flux of the [Ne V] line is $2 \times 10^{-15} \mathrm{erg} \mathrm{cm}^{-2} \mathrm{~s}^{-1}$ (Goulding \& Alexander 2009), which corresponds to a luminosity of $1.8 \times 10^{37} \mathrm{erg} \mathrm{s}^{-1}$ assuming a distance of $8.58 \mathrm{Mpc}$, in very good agreement with the $L_{\mathrm{Bol}}$ estimate.

We also find evidence for a break in the X-ray spectrum of M51b at 2-7 keV; however, since there may still be unresolved $\mathrm{X}$-ray binaries in the nucleus of M51b that could mimic this spectral shape, we cannot draw strong conclusions about this pertaining to the nature of the X-ray emission from the nucleus.

The luminosities and/or accretion rates inferred for the accreting SMBHs in the M51 system are lower than that predicted by galaxy merger simulations, especially considering M51 is rich in molecular gas $\left(2 \times 10^{9} M_{\odot}\right.$, e.g., Schirm et al. 2017; Schuster et al. 2007). Van Wassenhove et al. (2012) predict that for a 1:2 spiral-spiral merger, both AGNs should exhibit bolometric luminosities of $>10^{41} \mathrm{erg} \mathrm{s}^{-1}$ for the entire merger period. Even for a 1:2 elliptical-spiral merger, the secondary galaxy (in this case M51b) should exhibit $L_{\mathrm{Bol}}$ $\sim 10^{42} \mathrm{erg} \mathrm{s}^{-1}$ or higher for the entire merger. This is clearly not the case for M51.

Dual AGN activity occurs mainly at small separations ( $<10$ kpc; e.g., Satyapal et al. 2014; Fu et al. 2018), following the second and subsequent pericenter passages. Simulations have found that M51b has passed through M51a at least twice (Salo \& Laurikainen 2000). The angular separation between the nuclei of the two galaxies is 4.4 arcmin, which corresponds to a projected separation of $11 \mathrm{kpc}$ at $8.58 \mathrm{Mpc}$, although M51b is 
thought to be behind M51a, so the actual separation may be larger. Indeed, dynamical modeling suggests that the pericenter passage was $\sim 25 \mathrm{kpc}$ (Salo \& Laurikainen 2000), which, if this corresponds to the second pericenter passage, implies that M51 is in a relatively early stage of its merger compared to the other systems modeled (e.g., Hibbard \& Mihos 1995; Privon et al. 2013). Since dual AGNs with closer separations have higher Eddington ratios, the low Eddington ratios that we derive support the fact that we are observing the early stages of the merger. Also, there is likely to be considerable variability in the SMBH accretion rates that may be related to the merger, such as that observed in one of the dual AGNs in ESO 509IG066 (Kosec et al. 2017).

The inferred star formation history of M51 may also provide clues. These studies have found that the star formation rate of M51a peaked 1000-500 Myr ago at $\sim 10 M_{\odot} \mathrm{yr}^{-1}$, coinciding with the second-to-last encounter. The star formation rate over the past $100 \mathrm{Myr}$, during which the most recent encounter occurred, is much lower $\left(\sim 2 M_{\odot} \mathrm{yr}^{-1}\right.$, Mentuch Cooper et al. 2012; Eufrasio et al. 2017). If SMBH growth occurs simultaneously with star formation, this implies that the dual AGNs were more active during the encounter 400-500 Myr ago.

\subsection{The Ultraluminous X-Ray Source Population of the M51 Galaxies}

The ULXs in M51 have been extensively studied in previous works, both at X-ray wavelengths (e.g., Dewangan et al. 2005; Yoshida et al. 2010) and longer wavelengths (e.g., Terashima et al. 2006; Heida et al. 2014). Here, we have presented the first systematic study of these sources at hard X-ray wavelengths, afforded by a long exposure with NuSTAR. All ULXs studied so far with NUSTAR that have sufficient signal-to-noise broadband data show remarkably similar spectral shapes (Koliopanos et al. 2017; Pintore et al. 2017; Walton et al. $2018 \mathrm{~b}$ ), the most notable feature being a spectral turnover below $10 \mathrm{keV}$ (e.g., Bachetti et al. 2013). This feature is not seen in the X-ray spectra of any sub-Eddington accreting black holes (McClintock \& Remillard 2006).

The broadband ULX spectral shape is generally interpreted as the superposition of one or more disk-like components, in combination with a high-energy tail. ULXs that exhibit this behavior include the known neutron-star accretors such as M82 X-2 (albeit the spectral turnover has only been observed in the pulsed emission; Brightman et al. 2016), NGC 7793 P13 (Walton et al. 2018a), and NGC 5907 ULX1 (Walton et al. 2018b). For the neutron stars, the high-energy tail appears to be associated with the pulsed emission and therefore with emission from the accretion column that rotates with the neutron star (Walton et al. 2018b). Therefore, identifying a spectral turnover in other ULXs possibly identifies them as candidate super-Eddington accretors that are potentially powered by neutron stars.

While the ULXs in M51 are faint, and the signal to noise in the NUSTAR data is not as high as the sources presented in Walton et al. (2018b), we have found statistically significant evidence for a spectral turnover in three sources: ULX5, ULX7, and ULX9 at $2.6 \sigma, 3 \sigma$, and $>3 \sigma$ confidence respectively. Interestingly, only ULX8, already known to be powered by a neutron star, does not show evidence for a turnover. Nevertheless, the spectrum of ULX8 is consistent with a cutoff as low as $6 \mathrm{keV}$ at $90 \%$ confidence and a turnover was identified in a
Chandra observation when the source was observed at a higher flux (Brightman et al. 2018). This spectral turnover is also observed in other star-forming galaxies observed with NUSTAR, which are likely to be dominated by the emission from ULXs (Wik et al. 2014; Lehmer et al. 2015; Yukita et al. 2016). We note that a general presentation of extranuclear point sources observed by $N U S T A R$ in nearby galaxies is presented in Vulic et al. (2018).

We have also tested disk models for these sources, specifically a multicolor disk blackbody model with a free radial temperature profile index. While for a standard thin accretion disk this parameter is expected to be 0.75 , lower values have been measured in the spectra of ULXs which are expected from slim accretion disks (Abramowicz et al. 1988; Watarai et al. 2000; Poutanen et al. 2007). However, for only one of our sources are the parameters of this model well constrained, for ULX5. Here, the radial temperature profile is $0.6_{-0.1}^{+0.2}$, which is consistent with either a standard accretion disk and a slim disk. For ULX3, the radial temperature index is constrained to be $>0.7$, which would rule out a slim disk scenario.

Some ULX candidates have been found to be background AGNs in the past (e.g., Gutiérrez 2013). We can calculate the expected number of background extragalactic sources within the area of M51 using the X-ray number counts derived from $\mathrm{X}$-ray surveys. At $0.5-10 \mathrm{keV}$ fluxes $>10^{-13} \mathrm{erg} \mathrm{cm}^{-2} \mathrm{~s}^{-1}$, the $\mathrm{X}$-ray source number density is $20 \mathrm{deg}^{-2}$ (Georgakakis et al. 2008). Given an area of approximately $0.02 \mathrm{deg}^{2}$ subtended by M51, the expected number of background sources within the galaxy is 0.4 . The Poisson probability that one of the ULXs in M51 is a background source is therefore 0.27 , and the probability that more than one is a background source is $\leqslant 0.05$. However, most of the ULXs are located in the spiral arms, making their association with M51 more likely. ULX5 and ULX9 appear possibly offset from the galaxies' main structures, but for those sources we have found statistically significant evidence for a spectral turnover, so a background AGN scenario is disfavored since as stated above, these systems do not show this feature at energies below $10 \mathrm{keV}$. Finally, ULX7 and ULX8 both have probable stellar counterparts, all but ensuring their position within the galaxies (Terashima et al. 2006; Earnshaw et al. 2016).

While these deep NuSTAR data have allowed us to perform some basic spectral characterization of the ULXs in M51, the count rates in the Chandra or NUSTAR detectors are not high enough to conduct pulsation searches. Previous discoveries have required $\sim 10^{4}$ counts to detect pulsations (Bachetti et al. 2014; Fürst et al. 2016; Israel et al. 2017a, 2017b), whereas only a few hundred counts are observed from each source in each detector. We nevertheless carried out fast Fourier transform analyses on the light curves, but found no significant peaks.

\section{Summary and Conclusions}

We have presented a broadband X-ray spectral analysis of the AGN and off-nuclear point sources in the galaxies of M51 with simultaneous Chandra and deep NUSTAR observations. We have measured the intrinsic X-ray luminosities of the dual AGN with the highest fidelity yet, using the latest X-ray torus models to infer $L_{X}$ for the Compton-thick nucleus of M51a, and resolving the nucleus of M51b for the first time with Chandra. Both SMBHs have very low accretion rates $\left(\lambda_{\text {Edd }}<10^{-4}\right)$ 
considering that the galaxies are in the process of merging. We find that the covering factor of the torus in M51a is low, which agrees with the latest results on the local AGN obscured fraction that shows a low fraction at low luminosities. All of the ULXs we study show evidence for a spectral turnover, which appears to be ubiquitous when these sources are studied at high signal-to-noise ratios.

The authors thank the anonymous referee for the thorough and detailed review of our manuscript, which improved it. M. Baloković acknowledges support from the Black Hole Initiative at Harvard University, through the grant from the John Templeton Foundation. M.K. acknowledges support from NASA through ADAP award NNH16CT03C. D.M.A. acknowledges the Science and Technology Facilities Council (STFC) through grant ST/P000541/1. The work of D.S. was carried out at the Jet Propulsion Laboratory, California Institute of Technology, under a contract with NASA. A.Z. acknowledges funding from the European Research Council under the European Unions Seventh Framework Programme (FP/ 2007-2013)/ERC Grant Agreement n. 617001. Support for this work was provided by the National Aeronautics and Space Administration through Chandra Award Number GO7$18105 \mathrm{X}$ issued by the Chandra X-ray Center, which is operated by the Smithsonian Astrophysical Observatory for and on behalf of the National Aeronautics Space Administration under contract NAS8-03060. This work was also supported under NASA contract No. NNG08FD60C, and made use of data from the NUSTAR mission, a project led by the California Institute of Technology, managed by the Jet Propulsion Laboratory, and funded by the National Aeronautics and Space Administration. We thank the NuSTAR Operations, Software, and Calibration teams for support with the execution and analysis of these observations. This research has made use of the NUSTAR Data Analysis Software (NuSTARDAS) jointly developed by the ASI Science Data Center (ASDC, Italy) and the California Institute of Technology (USA).

Facilities: Chandra (ACIS), NuSTAR, Hale (DBSP).

\section{ORCID iDs}

M. Baloković (ib https://orcid.org/0000-0003-0476-6647

M. Koss (i) https://orcid.org/0000-0002-7998-9581

D. M. Alexander (ib https://orcid.org/0000-0002-5896-6313

A. Annuar (i) https://orcid.org/0000-0003-0387-1429

P. Gandhi (i) https://orcid.org/0000-0003-3105-2615

F. A. Harrison (1) https://orcid.org/0000-0003-2992-8024

B. Lehmer (1) https://orcid.org/0000-0003-2192-3296

M. C. Powell (1) https://orcid.org/0000-0003-2284-8603

B. Rangelov (1) https://orcid.org/0000-0002-9282-5207

T. P. Roberts (1) https://orcid.org/0000-0001-8252-6337

D. Stern (1) https://orcid.org/0000-0003-2686-9241

D. J. Walton (1) https://orcid.org/0000-0001-5819-3552

A. Zezas (i) https://orcid.org/0000-0001-8952-676X

\section{References}

Abramowicz, M. A., Czerny, B., Lasota, J. P., \& Szuszkiewicz, E. 1988, ApJ, 332,646

Annuar, A., Alexander, D. M., Gandhi, P., et al. 2017, ApJ, 836, 165

Arnaud, K. A. 1996, in ASP Conf. Ser. 101, Astronomical Data Analysis Software and Systems V, ed. G. H. Jacoby \& J. Barnes (San Francisco, CA: ASP), 17
Asmus, D., Gandhi, P., Hönig, S. F., Smette, A., \& Duschl, W. J. 2015, MNRAS, 454, 766

Bachetti, M., Harrison, F. A., Walton, D. J., et al. 2014, Natur, 514, 202 Bachetti, M., Rana, V., Walton, D. J., et al. 2013, ApJ, 778, 163

Baloković, M., Brightman, M., Harrison, F. A., et al. 2018, ApJ, 854, 42 Boorman, P. G., Gandhi, P., Baloković, M., et al. 2018, MNRAS, 477, 3775 Brightman, M., Baloković, M., Ballantyne, D. R., et al. 2017, ApJ, 844, 10 Brightman, M., Baloković, M., Stern, D., et al. 2015, ApJ, 805, 41

Brightman, M., Harrison, F., Walton, D. J., et al. 2016, ApJ, 816, 60

Brightman, M., Harrison, F. A., Fürst, F., et al. 2018, NatAs, 2, 312

Brightman, M., \& Nandra, K. 2011a, MNRAS, 413, 1206

Brightman, M., \& Nandra, K. 2011b, MNRAS, 414, 3084

Brightman, M., Silverman, J. D., Mainieri, V., et al. 2013, MNRAS, 433, 2485

Broos, P. S., Townsley, L. K., Feigelson, E. D., et al. 2010, ApJ, 714, 1582

Burlon, D., Ajello, M., Greiner, J., et al. 2011, ApJ, 728, 58

Cappellari, M. 2017, MNRAS, 466, 798

Cappellari, M., \& Emsellem, E. 2004, PASP, 116, 138

Cash, W. 1979, ApJ, 228, 939

Chen, Y.-P., Trager, S. C., Peletier, R. F., et al. 2014, A\&A, 565, A117

de Vaucouleurs, G., de Vaucouleurs, A., Corwin, H. G., Jr., et al. 1991, S\&T, 82,621

Dewangan, G. C., Griffiths, R. E., Choudhury, M., Miyaji, T., \& Schurch, N. J. 2005, ApJ, 635, 198

Dobbs, C. L., Theis, C., Pringle, J. E., \& Bate, M. R. 2010, MNRAS, 403, 625

Earnshaw, H. M., Roberts, T. P., Heil, L. M., et al. 2016, MNRAS, 456, 3840

Earnshaw, H. P., Roberts, T. P., \& Sathyaprakash, R. 2018, MNRAS, 476, 4272

Ehle, M., Pietsch, W., \& Beck, R. 1995, A\&A, 295, 289

Elitzur, M., \& Shlosman, I. 2006, ApJL, 648, L101

Ellison, S. L., Patton, D. R., Mendel, J. T., \& Scudder, J. M. 2011, MNRAS, 418, 2043

Eufrasio, R. T., Lehmer, B. D., Zezas, A., et al. 2017, ApJ, 851, 10

Fu, H., Steffen, J. L., Gross, A. C., et al. 2018, ApJ, 856, 93

Fukazawa, Y., Iyomoto, N., Kubota, A., Matsumoto, Y., \& Makishima, K. 2001, A\&A, 374, 73

Fürst, F., Walton, D. J., Harrison, F. A., et al. 2016, ApJL, 831, L14

Gandhi, P., Horst, H., Smette, A., et al. 2009, A\&A, 502, 457

Georgakakis, A., Nandra, K., Laird, E. S., Aird, J., \& Trichas, M. 2008, MNRAS, 388, 1205

Gladstone, J. C., Roberts, T. P., \& Done, C. 2009, MNRAS, 397, 1836

Goulding, A. D., \& Alexander, D. M. 2009, MNRAS, 398, 1165

Gruppioni, C., Berta, S., Spinoglio, L., et al. 2016, MNRAS, 458, 4297

Gutiérrez, C. M. 2013, A\&A, 549, A81

Harrison, F. A., Craig, W. W., Christensen, F. E., Hailey, C. J., \& Zhang, W. W. 2013, ApJ, 770, 103

Heida, M., Jonker, P. G., Torres, M. A. P., et al. 2014, MNRAS, 442, 1054

Hernández-García, L., González-Martín, O., Masegosa, J., \& Márquez, I. 2014, A\&A, 569, A26

Hernquist, L. 1989, Natur, 340, 687

Hibbard, J. E., \& Mihos, J. C. 1995, AJ, 110, 140

Ho, L. C., Greene, J. E., Filippenko, A. V., \& Sargent, W. L. W. 2009, ApJS, 183,1

Hopkins, P. F., Hernquist, L., Cox, T. J., et al. 2005, ApJ, 630, 705

Israel, G. L., Belfiore, A., Stella, L., et al. 2017a, Sci, 355, 817

Israel, G. L., Papitto, A., Esposito, P., et al. 2017b, MNRAS, 466, L48

Iwasawa, K., \& Taniguchi, Y. 1993, ApJL, 413, L15

Kaaret, P., Feng, H., \& Roberts, T. P. 2017, ARA\&A, 55, 303

Kato, S., Fukue, J., \& Mineshige, S. (ed.) 1998, Black-hole Accretion Disks

Kocevski, D. D., Brightman, M., Nandra, K., et al. 2015, ApJ, 814, 104

Koliopanos, F., Vasilopoulos, G., Godet, O., et al. 2017, A\&A, 608, A47

Komossa, S., Burwitz, V., Hasinger, G., et al. 2003, ApJL, 582, L15

Kormendy, J., \& Ho, L. C. 2013, ARA\&A, 51, 511

Kosec, P., Brightman, M., Stern, D., et al. 2017, ApJ, 850, 168

Koss, M., Trakhtenbrot, B., Ricci, C., et al. 2017, ApJ, 850, 74

Koss, M. J., Glidden, A., Baloković, M., et al. 2016, ApJL, 824, L4

Kuntz, K. D., Long, K. S., \& Kilgard, R. E. 2016, ApJ, 827, 46

Lansbury, G. B., Stern, D., Aird, J., et al. 2017, ApJ, 836, 99

Lanzuisi, G., Civano, F., Elvis, M., et al. 2013, MNRAS, 431, 978

Lanzuisi, G., Ranalli, P., Georgantopoulos, I., et al. 2015, A\&A, 573, A137

Lehmer, B. D., Eufrasio, R. T., Markwardt, L., et al. 2017, ApJ, 851, 11

Lehmer, B. D., Tyler, J. B., Hornschemeier, A. E., et al. 2015, ApJ, 806, 126

Levenson, N. A., Krolik, J. H., Życki, P. T., et al. 2002, ApJL, 573, L81

Liu, J.-F., \& Bregman, J. N. 2005, ApJS, 157, 59

Liu, Q. Z., \& Mirabel, I. F. 2005, A\&A, 429, 1125

Lusso, E., Comastri, A., Simmons, B. D., et al. 2012, MNRAS, 425, 623 
Madsen, K. K., Harrison, F. A., Markwardt, C. B., et al. 2015, ApJS, 220, 8 Marconi, A., Risaliti, G., Gilli, R., et al. 2004, MNRAS, 351, 169

McClintock, J. E., \& Remillard, R. A. 2006, in Black Hole Binaries, ed W. H. G. Lewin \& M. van der Klis (Cambridge: Cambridge Univ. Press), 157

McQuinn, K. B. W., Skillman, E. D., Dolphin, A. E., Berg, D., \& Kennicutt, R. 2016, ApJ, 826, 21

Mentuch Cooper, E., Wilson, C. D., Foyle, K., et al. 2012, ApJ, 755, 165

Messier, C. 1781, in Connoissance des Temps ou des Mouvements Célestes, 227

Murphy, K. D., \& Yaqoob, T. 2009, MNRAS, 397, 1549

Nelson, C. H., \& Whittle, M. 1995, ApJS, 99, 67

Oh, K., Koss, M., Markwardt, C. B., et al. 2018, ApJS, 235, 4

Palumbo, G. G. C., Fabbiano, G., Trinchieri, G., \& Fransson, C. 1985, ApJ, 298, 259

Pintore, F., Zampieri, L., Stella, L., et al. 2017, ApJ, 836, 113

Poutanen, J., Lipunova, G., Fabrika, S., Butkevich, A. G., \& Abolmasov, P. 2007, MNRAS, 377, 1187

Privon, G. C., Barnes, J. E., Evans, A. S., et al. 2013, ApJ, 771, 120

Querejeta, M., Schinnerer, E., García-Burillo, S., et al. 2016, A\&A, 593, A118

Rampadarath, H., Soria, R., Urquhart, R., et al. 2018, MNRAS, 476, 2876

Reines, A. E., \& Volonteri, M. 2015, ApJ, 813, 82

Ricci, C., Bauer, F. E., Treister, E., et al. 2017a, MNRAS, 468, 1273

Ricci, C., Paltani, S., Awaki, H., et al. 2013, A\&A, 553, A29

Ricci, C., Trakhtenbrot, B., Koss, M. J., et al. 2017b, Natur, 549, 488

Roberts, T. P. 2007, Ap\&SS, 311, 203

Salo, H., \& Laurikainen, E. 2000, MNRAS, 319, 377

Sanders, D. B., Soifer, B. T., Elias, J. H., et al. 1988, ApJ, 325, 74

Satyapal, S., Ellison, S. L., McAlpine, W., et al. 2014, MNRAS, 441, 1297

Schirm, M. R. P., Wilson, C. D., Kamenetzky, J., et al. 2017, MNRAS, 470, 4989

Schlegel, E. M., Jones, C., Machacek, M., \& Vega, L. D. 2016, ApJ, 823, 75
Schuster, K. F., Kramer, C., Hitschfeld, M., Garcia-Burillo, S., \& Mookerjea, B. 2007, A\&A, 461, 143

Shakura, N. I., \& Sunyaev, R. A. 1973, A\&A, 24, 337

Shemmer, O., Brandt, W. N., Netzer, H., Maiolino, R., \& Kaspi, S. 2006, ApJL, 646, L29

Smith, B. J., Swartz, D. A., Miller, O., et al. 2012, AJ, 143, 144

Stauffer, J. R. 1982, ApJ, 262, 66

Stobbart, A.-M., Roberts, T. P., \& Wilms, J. 2006, MNRAS, 368, 397

Swartz, D. A., Soria, R., Tennant, A. F., \& Yukita, M. 2011, ApJ, 741, 49

Terashima, Y., Inoue, H., \& Wilson, A. S. 2006, ApJ, 645, 264

Terashima, Y., \& Wilson, A. S. 2001, ApJ, 560, 139

Terashima, Y., \& Wilson, A. S. 2004, ApJ, 601, 735

Theis, C., \& Spinneker, C. 2003, Ap\&SS, 284, 495

Toomre, A., \& Toomre, J. 1972, ApJ, 178, 623

Trakhtenbrot, B., Ricci, C., Koss, M. J., et al. 2017, MNRAS, 470, 800

Urquhart, R., \& Soria, R. 2016, ApJ, 831, 56

Van Wassenhove, S., Volonteri, M., Mayer, L., et al. 2012, ApJL, 748, L7

Vasudevan, R. V., Brandt, W. N., Mushotzky, R. F., et al. 2013, ApJ, 763, 111

Vulic, N., Hornschemeier, A. E., Wik, D. R., et al. 2018, ApJ, 864, 150

Wada, K. 2015, ApJ, 812, 82

Walton, D. J., Fürst, F., Harrison, F. A., et al. 2018a, MNRAS, 473, 4360

Walton, D. J., Fürst, F., Heida, M., et al. 2018b, ApJ, 856, 128

Walton, D. J., Roberts, T. P., Mateos, S., \& Heard, V. 2011, MNRAS, 416, 1844

Watarai, K.-y., Fukue, J., Takeuchi, M., \& Mineshige, S. 2000, PASJ, 52, 133

Wik, D. R., Lehmer, B. D., Hornschemeier, A. E., et al. 2014, ApJ, 797, 79

Winter, L. M., Mushotzky, R. F., \& Reynolds, C. S. 2006, ApJ, 649, 730

Woo, J.-H., \& Urry, C. M. 2002, ApJ, 579, 530

Xu, W., Liu, Z., Gou, L., \& Liu, J. 2016, MNRAS, 455, L26

Yoshida, T., Ebisawa, K., Matsushita, K., Tsujimoto, M., \& Kawaguchi, T. 2010, ApJ, 722, 760

Yukita, M., Hornschemeier, A. E., Lehmer, B. D., et al. 2016, ApJ, 824, 107 